\title{
Dynamics and thermodynamics of polymer glasses TOPICAL REVIEW
}

\author{
D. Cangialosi \\ IOP Publishing, Dirac House, Temple Back, Bristol BS1 6BE, UK \\ E-mail: custserv@iop.org
}

\begin{abstract}
.
The fate of matter when decreasing the temperature at constant pressure is that of passing from gas to liquid and subsequently from liquid to crystal. However, a class of materials can exist in the amorphous phase below the melting temperature. On cooling such materials a glass is formed, that is, a material with the rigidity of a solid but exhibiting no long-range order. The study of the thermodynamics and dynamics of glass-forming systems is subjected to continuous research. Within the wide variety of glass formers, an important sub-class is represented by glass forming polymers. The presence of chain connectivity and, in some cases, conformational disorder are unfavourable factors from the point of view of crystallization. Furthermore, many of them, such as amorphous thermoplastics, thermosets and rubbers, are widely employed in many applications. In this Review, the peculiarities of the thermodynamics and dynamics of glass forming polymers are discussed with particular emphasis on those topics currently subject of debate. In particular, the following aspects will be reviewed in the present work: i) the connection between the pronounced slowing down of glassy dynamics on cooling toward the glass transition temperature $\left(T_{g}\right)$ and the thermodynamics; ii) the fate of the dynamics and thermodynamics below $T_{g}$. Both aspects are reviewed in light of the possible presence of a singularity at a finite temperature with diverging relaxation time and zero configurational entropy. In this context, the specificity of glass forming polymers is emphasized.
\end{abstract}

\section{Introduction}

Structurally disordered materials are widely encountered in nature and employed in a variety of technologically relevant applications. Among disordered systems an interesting class from a scientific point of view is represented by glass forming materials $[1,2]$. These systems, below the melting temperature $\left(T_{m}\right)$, exist in the disordered amorphous state rather than falling to the most stable thermodynamic state, that is, the most stable crystalline form. As such the glassy state, despite being a wellknown state of matter, is not included among states of aggregation in the classical thermodynamic meaning. Glass forming materials are by definition those systems that remain amorphous (in the so-called supercooled state) over extremely long time 
scales without crystallizing. The glass-forming ability of different class of materials depends on a number of parameters. Among the variety of glass forming systems, a considerable number of polymers represents an important class. These systems exhibit complex crystallization behaviour due to the presence of chain connectivity and, in some cases, conformational irregularity. Hence, due to the great variety of non-crystallizable polymers as well as their wide use in a number of applications in the amorphous state (e.g. rubbers, amorphous thermoplastics and thermosets), glass forming polymers have been intensively studied since the appearance of the first synthetic polymers dating back to the beginning of the last century [3, 4].

In this Review, after highlighting the well accepted features of the dynamics and thermodynamics of glass forming polymers, included the methods to characterize them, we devote special attention to those specific topics that are currently hotly debated in an attempt of understanding the nature of the glassy state. Special attention is devoted to the current understanding of polymer glasses, though, relying on the universality of the behaviour of glasses of any nature, the connection with non-polymeric glass formers will be often recalled. In particular, the following aspects will be reviewed: i) the connection between dynamics and thermodynamics and, within this context, the specificity of polymers and other complex glass-forming materials; ii) the recent developments reporting on the fate of the dynamics and thermodynamics when going down the energy landscape, that is, when the metastable equilibrium is probed at relatively low values of the energy.

\section{Dynamics and thermodynamics of glass-forming polymers: established facts}

\subsection{Thermodynamic aspects}

As discussed in the previous section, two main events lead to the formation of a glass: i) the avoidance of crystallization when cooling down below the $T_{m}$; ii) the liquid to glass transition occurring when cooling down the supercooled liquid across the $T_{g}$. This is sketched in Fig. 1, where a schematic representation of the thermodynamics of glass forming systems at constant pressure is provided.

From a thermodynamic point of view, albeit not in the lowest (absolute) energy minimum, non-crystallizable supercooled liquids are classified as metastable, that is, they are stable when small perturbations are applied [1, 2]. This is illustrated in Fig. 1 where the free energy in the configurational space is schematically depicted. Due to its location in a free energy minimum, the configuration corresponding to the supercooled liquid can persist over infinitely large time scales and the principles of classical equilibrium thermodynamics can be generally applied.

On further cooling, a jump in second order thermodynamic properties - such as specific heat, compressibility and coefficient of thermal expansion - of the supercooled liquid is observed. The temperature at which such jump is observed is commonly 


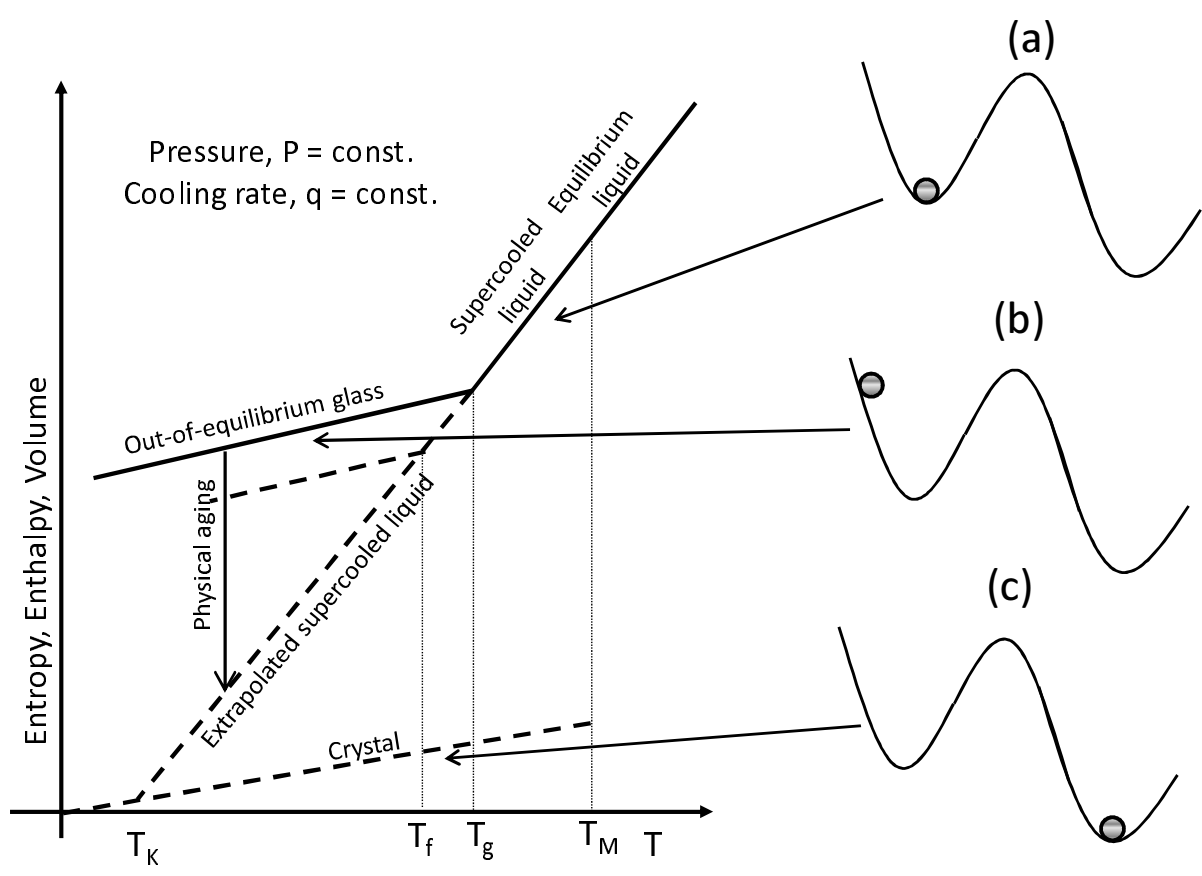

Figure 1. (Left side) Schematic representation of the temperature dependence of thermodynamic properties in glass forming systems at constant pressure. Here the following temperatures are indicated: i) $T_{M}$, the melting temperature; ii) $T_{g}$, the temperature at which the supercooled liquid transforms into a glass at the cooling rate of the experiment; iii) $T_{f}$, that is, the temperature at which a glass with given structure, e.g. after aging at a temperature below $T_{g}$, would be at equilibrium; iv) $T_{K}$, the Kauzmann temperature, that is, the temperature at which the entropy of the supecooled liquid equals that of the corresponding crystal. (Right side) Stability plots for (a) the metastable supercooled liquid; (b) the out-of-equilibrium glass; and (c) the stable crystal

addressed as the liquid to glass transition temperature $\left(T_{g}\right)$ (see Fig. 1). Below this temperature a glass is obtained, that is a system with the mechanical properties of a solid but exhibiting the typical structural features of a disordered liquid.

The typical signature of the liquid-glass transition, namely the jump in second order thermodynamic properties, is reminiscent of an Ehrenfest thermodynamic transition [2]. However our current knowledge of the glass transition indicates that the liquid to glass transition does not fulfil the requirements of a thermodynamic transition in a strict sense. The most straightforward indication of the absence of an Ehrenfest second order transition is the cooling rate dependence of the $T_{g}$. As will be seen in the following section, this is due to the intimate link of the cooling rate dependent $T_{g}$ with the molecular mobility in the glass former. This aspect emphasizes the kinetic nature of the glass transition. A further indication of its kinetic nature is the breadth of the glass 
transition, generally encompassing a rather large temperature interval. Finally, from the point of view of classical thermodynamics, an Ehrenfest second order transition would fulfil the relation:

$$
\Pi=\frac{1}{V T} \frac{\Delta C_{p} \Delta k}{(\Delta \alpha)^{2}}=1
$$

where $\Delta C_{p}, \Delta k$ and $\Delta \alpha$ are the specific heat, the compressibility and the coefficient of thermal expansion jumps at the transition respectively. It is well documented [2] that at the glass transition the previous relation does not hold and $\Pi$, which in this context is known as the Prigogine-Defay ratio [5], is larger than unity.

Despite the evidences for the non-thermodynamic nature of the glass transition, this is still a matter of open debate. This originates for the work presented long ago by Kauzmann [6]. Analysing thermodynamic data of many glass formers, including polymers, Kauzmann noticed that, since the specific heat of supercooled liquids is larger than that of the corresponding crystal, there will be a temperature where the first order thermodynamic properties of the glass will equal those of the crystal. In the analysis by Kauzmann such a temperature, now known as the Kauzmann temperature $\left(T_{K}\right)$, is generally larger than zero kelvin. This is schematically depicted in Fig. 1, where a given thermodynamic property is displayed for a generic glass former in the supercooled liquid as well as in the glassy and crystalline state. Kauzmann's observation has fascinated many scientists involved in studies on the glassy state, since this would mean that a disordered system (the glass) and an ordered one (the corresponding crystal) exhibit identical entropies at $T_{K}>0$, a scenario hard to imagine considering the microscopic view of the entropy. Hence such a possibility, never experimentally tested due to the occurrence of the kinetic glass transition, has revitalized the scientific debate on whether en Ehrenfest second order thermodynamic transition exists at $T_{K}$ in vitrifying systems $[7,8]$.

From a thermodynamic point of view, glasses belong to the category of nonequilibrium systems (Fig. 1b). This means that systems in the glassy state spontaneously evolve with time toward the closest free energy minimum (see Fig. 1), that is, the corresponding metastable supercooled liquid. This phenomenon, known as physical aging, has immense scientific as well as technological implications and has been deeply investigated since the pioneering work of Simon [9] and more recently Kovacs [10] and Struik [11]. The most common way to enter the physical aging regime is to cool down the supercooled melt through the $T_{g}$ corresponding to a given cooling rate into the glassy state. Generally the nominal $T_{g}$ of a glass former corresponds to conventional laboratory cooling rates (e.g. by differential scanning calorimetry (DSC)), that is of the order of several kelvin per minute). Since the work of Kovacs [10] and Struik [11], huge amount of work has been dedicated to monitoring of the evolution of thermodynamic properties (or related to) toward the metastable equilibrium. In glassy polymers, the time evolution of the volume, the enthalpy as well as other properties sensitive to the glass thermodynamic state, have been investigated in the physical aging regime. The 
interested reader is addressed to those works where such studies are reviewed $[12,13,14]$. This kind of experiments allows extracting a typical time scale of glassy equilibration. Such aspect, dealing with the dynamics in the glassy state, will be described in detail in the following section of the Review. Within this subsection, it is worth noticing that physical aging of glasses allows going down in the energy landscape. A common way to define the thermodynamic state of a glass is that based on the fictive temperature $\left(T_{f}\right)$ long ago introduced by Tool [15], that is, the temperature at which a glass in a certain thermodynamic state would be at equilibrium (see Fig. 1). The lower the $T_{f}$ is, the lower in the energy landscape the glass (and closer to the Kauzmann singularity) is located. The ability of going down the landscape means that, at least until a certain extent dictated by the inevitably limited explorable aging times, those issues relating with the fate of the thermodynamics below the laboratory $T_{g}$ can be investigated. In particular, whether thermodynamic properties at the metastable equilibrium follow the trend observed in the supecooled state above $T_{g}$ can be experimentally verified. This assumes great importance within the context of the possible existence of a second order thermodynamic transition at $T_{K}$.

\subsection{Dynamic aspects}

The viscosity of a liquid above the melting temperature generally exhibits Arrhenius temperature dependence. Conversely a liquid cooled down below the melting temperature is characterized by a dramatic increase of the viscosity with decreasing temperature. Such an increase in viscosity implies the concomitant slowing down of the typical relaxation time of the associated spontaneous fluctuations. The phenomenon related to such relaxation time is normally called the $\alpha$ process. When the typical relaxation time of spontaneous fluctuations is of the order of the observation time (for instance the inverse of the cooling rate) the system is not able to maintain the (metastable) equilibrium state and a glass is formed. As discussed in the previous subsection, this occurs in a relatively narrow range around the $T_{g}$.

Among the numerous approaches successfully accounting for the steep increase of the relaxation time of the $\alpha$ process with decreasing temperature, historically the most employed by far is that presented around the 30ies by several authors. This the socalled Vogel-Fulcher-Tammann (VFT) equation that expresses the relaxation time as $[16,17,18]$ :

$$
\tau=\tau_{0} \exp \left(\frac{B}{T-T_{0}}\right)
$$

where $T_{0}$ is the so-called Vogel temperature, $B$ the Vogel activation energy and $\tau_{0}$ a pre-exponential factor. The VFT equation insinuates divergence of the relaxation time at $T_{0}$. However, the presence of a singularity at $T_{0}$ is based on the mere extrapolation of the relaxation time associated to glassy dynamics below the $T_{g}$. This is due to the fact that the intervening glass transition prevents measuring on reasonably short time scales 
the relaxation time of the equilibrium glass. Hence, the vast majority of experiments are performed above the $T_{g}$ of the glass. In this regime the validity of the VFT equation is proved in a wide temperature range. When very large temperature intervals are considered more than one VFT equation may be required to fit experimental data [19]. An equation, specifically developed for polymeric glass formers and equivalent to the VFT one, was proposed by Williams, Landel and Ferry (WLF) [20].

The rapidity of variation of $\tau$ is generally quantified by the steepness index $m$, that is, the derivative of the decimal logarithm of $\tau$ to the inverse temperature [21]:

$$
m=\left.\frac{d \log (\tau)}{d\left(T_{g} / T\right)}\right|_{T_{g}}
$$

The steepness index is a measure of the so-called "kinetic fragility". Large values of $m$ indicate pronounced temperature dependence of $\tau$. In such a case the glass former is addressed as fragile. This is the case of the vast majority of glass forming polymers exhibiting values of $m$ close to 100 and, in some cases, even larger. Conversely socalled strong glass formers exhibit low values of $m$. Among them network glasses are generally the strongest glass formers, with $m$ as low as 20 [22]. Apart from the steepness index, other metrics have been employed to describe the kinetic fragility of glass forming systems. This will be discussed in subsequent sections of the manuscript (Sections 4.3 and 4.4), in relation with the debate on the connection between the kinetic fragility, on the one hand, and thermodynamic and other properties, on the other.

An important aspect of the dynamics of glass forming liquids is its non-exponential nature. In particular, the decay of any function $(\phi(t))$ related to the spontaneous fluctuations of the $\alpha$ process (correlation, relaxation, retardation function etc.) appears to be highly stretched and is generally fitted empirically fitted by the so-called Kohlrausch-Williams-Watt (KWW) equation [23]:

$$
\phi(t)=\exp \left[-\left(\frac{t}{\tau}\right)^{\beta}\right]
$$

where $\beta$, the stretching exponent, is commonly smaller than the unity. The origin of the non-exponential nature of the dynamics in glass forming liquids is a hotly debated topic. This can in fact be the result of either the presence of spatially heterogeneous regions each relaxing exponentially $[24,25,26]$ or the intrinsic non-exponential nature of each (homogeneous) structural domain [27, 28]. The current knowledge indicates that both scenarios contribute to the stretching of the dynamic response [29]. In particular, the presence of spatial heterogeneities is proved by a number of experiments including nuclear magnetic resonance (NMR) [30, 24, 31, 26], dielectric hole burning [32], solvation dynamics [33] and photobleaching [34]. On the other hand the intrinsic non-exponential nature of the relaxation has been evidenced by neutron scattering experiments and molecular dynamics simulations [28, 35]. Specifically for polymers it has been shown that the degree of non-exponentiality is intimately linked to the height of the in-chain 
bond torsional barrier [35]. When such a barrier is allowed to increase in simulated model glass forming polymers, the stretching of the relaxational response becomes larger.

In highlighting the two main features characterizing the $\alpha$ relaxation of supercooled liquids, that is the non-Arrhenius temperature dependence and the non-exponential behaviour, we notice that a connection between these two aspects has been attempted [22], though results are not convincingly pointing toward a one-to-one relation between them $[29,36]$.

A way to explore the fate of the dynamics below the nominal $T_{g}$ consists in following the kinetics of recovery of equilibrium of the glass in the physical aging regime. This can be performed monitoring the time evolution of thermodynamic properties, such as the enthalpy or the volume, or properties related to the thermodynamics of the glass $[10,12,13,14]$. However, it is worth emphasizing that, despite the common view univocally associating the time scale of the recovery of equilibrium to that of spontaneous molecular fluctuations in the glass, the two time scales are conceptually different. In particular, the former time scale provides information on the recovery of equilibrium when the system has been perturbed beyond the linear regime [14]. Hence, information on the dynamics obtained in this regime are not strictly representative of the behaviour of spontaneous fluctuations, which can be rigorously probed only applying a perturbation in the linear regime. Notwithstanding this conceptual difference, in bulk glass formers a direct correlation between the rate of approach to equilibrium in the aging regime and that of molecular mobility has been successfully established since the pioneering work of Kovacs [10]. More recent works indicate that this is case when the cooling rate dependence of the $T_{g}$ is explored [37, 38, 39]. In particular, it has been shown that the parameters $T_{0}$ and $B$ of the VFT equation able to describe the relaxation time of the $\alpha$ process are also suitable to fit the cooling rate dependence of $T_{g}$. In this case a direct correlation between the experimental observation time and that of the intrinsic molecular mobility is established.

Beyond the motion responsible for glassy dynamics, glass-forming polymers also exhibit a range of different relaxational processes covering different length scales. Among them, a specific feature of the dynamics of polymers is the motion of the overall chain. This kind of motion will be not be discussed in the present Review, since it is not directly coupled to the thermodynamics of polymer glasses and, in particular, to the glass transition. As a matter of fact no molecular weight dependence of the thermodynamic properties, such as the specific heat or the coefficient of thermal expansion is experimentally found [40], whereas the $T_{g}$ is molecular weight independent above a certain limiting value $[41,42,43]$. Within the complex relaxational pattern of polymer glasses, localized motions, i.e. secondary relaxations, associated to pendant groups or main chain degrees of freedom are of considerable importance. Among them the localized rearrangement of one structural unit, named the Johari-Goldstein (JG) relaxation [44] is of interest in what concerns its possible connection to the $\alpha$ process [45]. Whatever the microscopic nature of these secondary motions, that involving the whole structural unit [44] or associated to some internal degrees of freedom [46], these generally 
exhibit typical relaxation times in the sub-second range even in the glassy state. As such, as will be discussed in this Review, they may cover significant importance in the physical aging regime. Short wave-length dynamics, namely that related to vibrational degrees of freedom are also of significant interest due to their connection with the thermodynamics and possibly with the dynamics of the $\alpha$ process too $[47,48]$.

\section{Experimental methods to probe the dynamics and thermodynamics of glasses}

This section, rather than describing in detail the techniques allowing to probe the dynamics and thermodynamics of glasses, aims to provide a general overview of the most employed methods. Particular attention is given to those measurements used in the topics developed in the present Review.

\subsection{Techniques probing the thermodynamics of glasses}

The thermodynamics of glass forming systems can be straightforwardly probed determining the temperature and pressure dependence of thermodynamic magnitudes, such as the volume or the enthalpy. The former is directly accessed by standard dilatometric techniques delivering the pressure-volume-temperature behaviour of the glass former [40]. The most common way to determine the temperature dependence of the volume of a specimen is to measure its length along a temperature ramp. In this way information on the linear coefficient of thermal expansion is achieved. Recently devices able to perform a characterization of the volume over a wide range of temperatures and pressures have been developed [49]. Precise determinations of the volume of a specimen are traditionally achieved by capillary dilatometry, generally based on the employment of mercury as dilating liquid [10]. In this kind of dilatometer the volume change is detected by a shift in the height of the dilating liquid in a capillary. Another widely employed method to measure the volume is that based on gradient columns. In this case two miscible solvents exhibiting specific volumes in the range expected for the material under investigation and where such material is insoluble, are filled in a column. The denser solvent is filled at the bottom of the column, whereas the less dense at the top. Thus along the column a density profile is established. Once a calibration of the density versus the vertical position in the column is established, the specific volume of a sample in a range between that of the two liquids in the column can be determined. Both the methods based on gradient columns and capillary dilatometry generally have the limitation that the volume can only be determined at atmospheric pressure conditions.

Methods based on calorimetry are extensively employed in the characterization of glass-forming systems. These are based on the way heat is exchanged between the material under investigation and the external world. Almost all calorimetric measurements are performed at atmospheric pressure and, therefore, the heat exchanged in a given experiment equals the variation in the enthalpy of the system. The 
absolute value of this thermodynamic function cannot be directly accessed but only its temperature variation, that is, the specific heat at constant pressure: $C_{p}=(\partial H / \partial T)_{P}$, is rather determined. Precise measurements of the latter require the use of calorimeters operating in (quasi)isothermal conditions. Adiabatic calorimeters belongs to such a class of instruments. To fulfil the requirement of (quasi)isothermal conditions, a recently developed method to measure the $C_{p}$ is that of modulated differential scanning calorimetry (MDSC) [50]. In comparison to standard differential scanning calorimetry (DSC), the superposition of a frequency modulated temperature program to the conventional heating/cooling ramp allows obtaining sufficiently large heat flows at (quasi)isothermal conditions [50, 51, 52].

In several instances, for what concerns the characterization of glasses thermodynamics standard DSC is generally employed [53]. In this case, the specific heat is obtained in non-isothermal conditions. In particular a heating/cooling rate is applied to the sample plus the pan where it is contained, and a reference, which is represented by an empty pan with mass very close to that containing the sample. The difference in the heat delivered to the sample and the reference provides information on the sample $C_{p}$. Due to the non-isothermal conditions, this may differ from the precise value by $10-15 \%$ depending on the calibration of the calorimeter and the applied heating/cooling rate. Within the context of glassy thermodynamics, standard DSC can be quantitatively exploited for a precise determination of: i) the jump of the specific heat at $T_{g}$, that is, the difference between the melt and glass specific heat; ii) the enthalpy recovered in the physical aging regime. An example of how this can be determined is presented in Fig. 2, where specific heat scans are displayed for different annealing times at $358 \mathrm{~K}$ for polystyrene (PS) [54]. Given the definition of the specific heat, the amount of recovered enthalpy can be evaluated from the difference of the area below the thermogram of the aged sample and that of the unaged one (so-called reference) [53]:

$$
\Delta H\left(T_{a}, t_{a}\right)=\int_{T_{x}}^{T_{y}}\left(C_{p}{ }^{a}(T)-C_{p}{ }^{u}(T)\right) d T
$$

where $C_{p}{ }^{a}(T)$ and $C_{p}{ }^{u}(T)$ are respectively the specific heat of the aged and unaged samples, and $T_{x}$ and $T_{y}$ are respectively temperatures well below and above the calorimetric $T_{g}$.

Apart from the determination of the volume and enthalpy, a number of other properties have been monitored to characterize the thermodynamics of glasses. This can be done if the temperature and pressure dependence of such properties mimics that of volume or enthalpy. Among them we can include the refractive index, the free volume by positron annihilation lifetime spectroscopy (PALS), properties related to the polarizability by dielectric methods, absorption in the infrared range, fluorescent spectroscopy and ellipsometry. The interested reader can consult those reviews for relevant works where these techniques are employed $[12,55,13,14]$. 


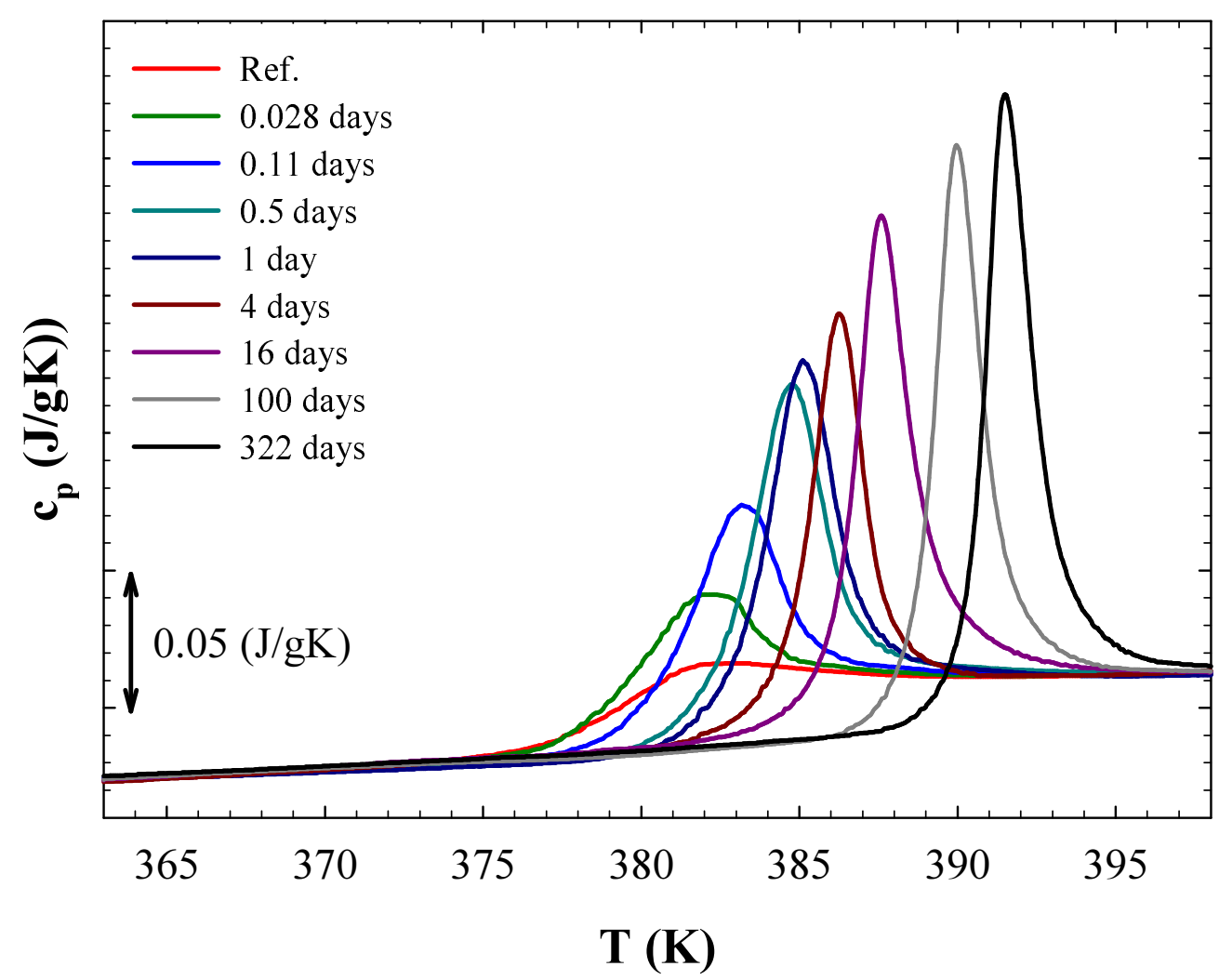

Figure 2. Specific heat as a function of temperature of PS aged at $363 \mathrm{~K}$ for the reported annealing time (reprinted with permission from ref. [54]).

\subsection{Techniques probing the dynamics of glasses}

Extensive studies have been performed in the past with the aim of characterizing the dynamics of glass-forming systems. Before entering the details of the commonly employed techniques, it is worth distinguishing them in two categories: i) those based on the application of a perturbation in the linear regime, that is, with amplitude smaller than that of spontaneous fluctuations; ii) methods where the dynamics of recovery of equilibrium after application of large perturbation to the system is monitored.

Regarding the former category, the combination of different techniques has nowadays allowed the determination of the typical relaxation time of glassy dynamics in a range comprised between picoseconds and several years. Furthermore some techniques provide spatial resolution, that is, the relaxation time is delivered for spontaneous fluctuations occurring within a length scale given by the experimental conditions.

Among the techniques only providing information on the time scale of spontaneous fluctuations, the most popular ones are broadband dielectric spectroscopy (BDS) [56], dynamic-mechanical [57] and specific heat (AC-calorimetry) spectroscopy [58, 59]. The most employed mode of operation of these techniques is based on frequency domain measurements. Here a sinusoidal field is applied to the sample. As a result a complex susceptibility is obtained: $\chi^{*}=\chi^{\prime}+i \chi^{\prime \prime}$. 
Dynamic-mechanical spectroscopy and AC-calorimetry are based on the same principles of BDS but with different applied perturbation. However, in contrast to BDS, the transmission of a mechanical field and that of thermal waves, in dynamicmechanical spectroscopy and AC-calorimetry respectively, is considerably more arduous to be performed at relatively high frequencies. The employment of modern equipments $[58,62,63,64]$ has allowed extending the upper frequency limit toward the $\mathrm{MHz}$ (or higher) regime.

Measurements in the time domain, mostly in dynamic-mechanical spectroscopy and BDS, are generally employed when the typical relaxation time of the glass is larger than seconds. In this context, dynamic-mechanical and dielectric measurements in the aging regime are vastly employed, since they allow monitoring the evolution of the typical relaxation time during equilibrium recovery. An example of such kind of characterization is presented in Fig. 4 for poly(vinyl chloride) (PVC) at $20{ }^{\circ} \mathrm{C}$, after cooling from 90 ${ }^{\circ} \mathrm{C}$ and different aging times [11]. As can be observed, the evolution of the mechanical compliance becomes increasingly slower during the course of aging implying a progressive increase of the relaxation time of the glass.

Methods delivering spatial information are based on scattering techniques $[65,66$, 67] and nuclear magnetic resonance (NMR) [31]. A common feature of the former category of techniques is that spatial resolution originates from the possibility of detecting scattered entities at different angles. According to the Bragg's law: $n \lambda=2 d$ $\sin \delta$; the length scale $d$ relevant to the measurement increases when decreasing the scattering angle $\delta$ (here $n$ is an integer and $\lambda$ the wavelength). The scattered entity can be neutrons, X-rays or photons in light scattering techniques. The employment of these techniques allows a more limited time scale interval in comparison to BDS. In particular, techniques based on neutron scattering (NS) have their upper bound in time scale at several hundreds nanoseconds. Light scattering techniques cover larger time scale intervals. However, due to the large wave length scale of light (of the order of several hundreds nanometers), density fluctuations are probed over volumes much larger than those relevant for glassy dynamics. The employment of new synchrotron radiation $\mathrm{X}$-ray sources has recently allowed measuring the dynamics with spatial resolution for 


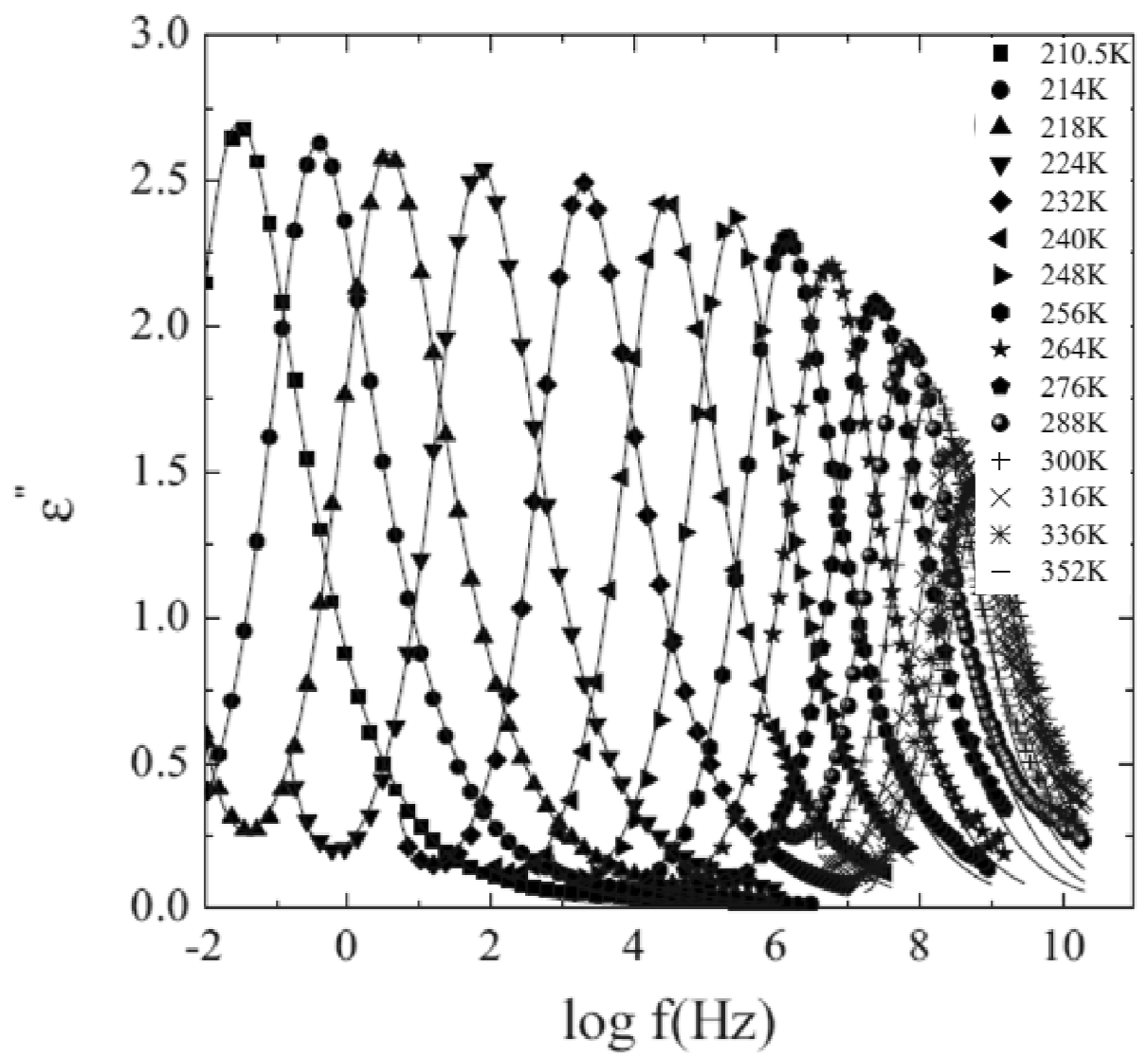

Figure 3. Loss part of the dielectric permittivity as a function of the decimal logarithm of the frequency for PVAc at different temperatures (reprinted with permission from ref. $[60])$.

time scales considerably larger than seconds. In this case the technique is called X-ray photon correlation spectroscopy (XPCS) $[68,69]$. In the case of NMR techniques, among the different aspects amenable to be investigated, these are capable of identifying the specific moieties undergoing a relaxation and the geometry of its motion (e.g. the angle of rotation) [31].

As anticipated, the previous methods deliver information on the time scale of spontaneous fluctuations in the glass former. A different approach is based on the application of large perturbations and the subsequent monitoring of equilibrium recovery. An example is that of following the isothermal evolution of a thermodynamic property after cooling the glass through its $T_{g}$, that is, in the physical aging regime $[70,71]$. From these kind of experiments a time scale of equilibration can be extracted. Similar information related to the efficiency of equilibration can be extracted from cooling rate dependent determination of the $T_{g}$. This similarity originates from the definition of $T_{g}$, that is, the temperature marking the equilibrium to out-of-equilibrium crossover. In both the monitoring of the time evolution of a thermodynamic property and the determination of cooling rate dependent $T_{g}$ a thermal perturbation beyond the 


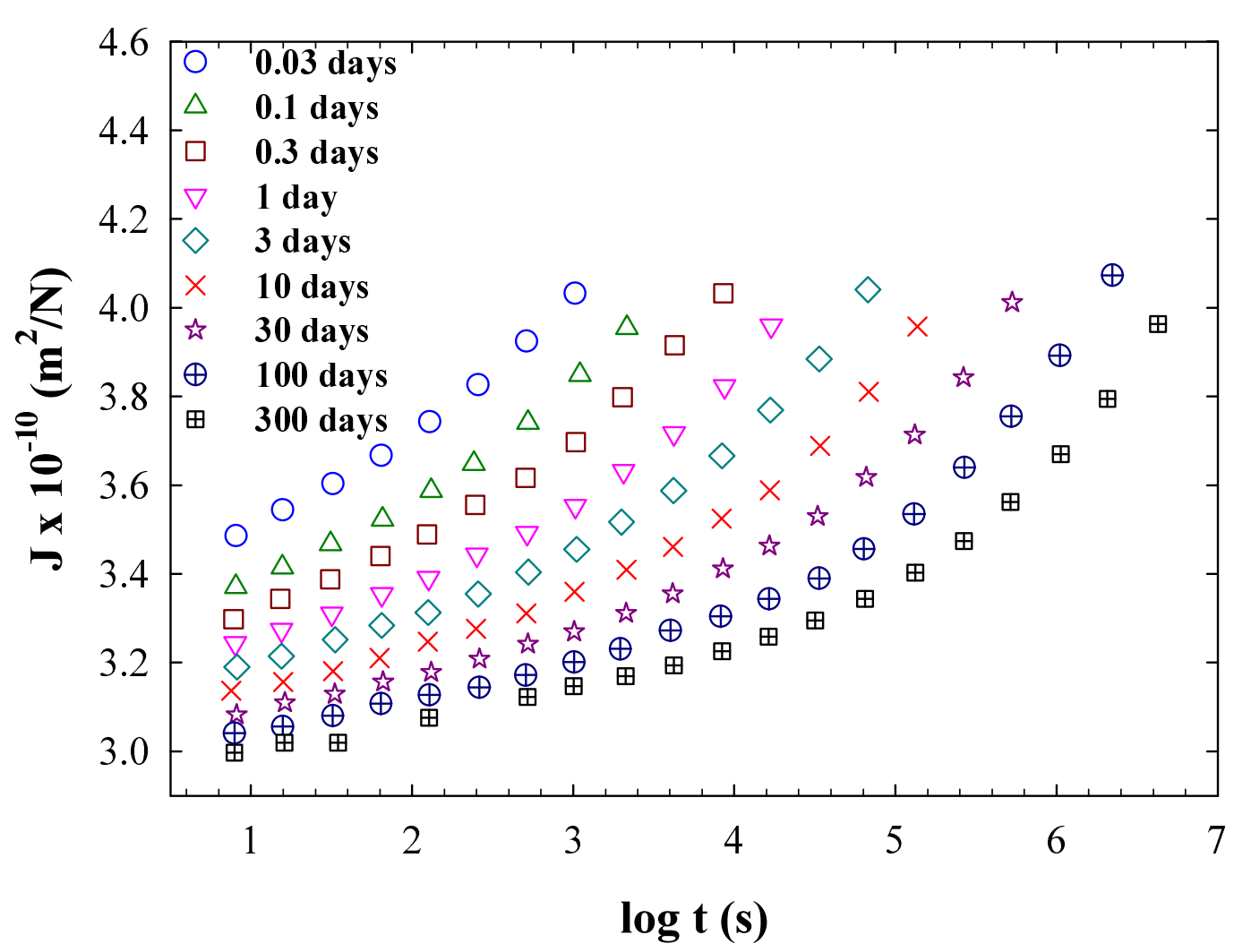

Figure 4. Mechanical compliance of PVC as a function of the decimal logarithm of the time measured by linear tensile creep experiments for different aging times after quenching from $90{ }^{\circ} \mathrm{C}$ to $20^{\circ} \mathrm{C}$ (adapted from Ref. [11]).

linear regime is applied. Other methods - based on following the kinetics of recovery are based on a mechanical or dielectric perturbation, or of any other magnitude amenable to be followed during equilibrium recovery - have received some attention in recent years $[32,72]$.

\section{Connection between dynamics and thermodynamics of glasses}

In this Review, we provide special attention to the thermodynamic description of the dynamics of glass formers. However, it is worth emphasizing that a considerable number of different approaches have been presented in the last decades. These generally range from exclusively kinetic pictures of glassy dynamics, such as the MCT [73] and kinetically constrained models [74], to those based on elastic models [75].

\subsection{Approaches based on the volume and the free volume}

The search for a thermodynamic explanation to the dynamics of glass-forming liquids dates back the beginning of systematic studies on the glass transition. The first approach in this sense, based on the connection of glassy dynamics with the free volume, was proposed by Doolittle in the early '50ies [76]. This approach relies on the fact that the 
volume of glasses in excess to that of the corresponding crystal must be associated to the presence of unoccupied space. Since then several models, all based on the idea that the rate of spontaneous fluctuations increases with the amount of empty space in the glass, were presented $[20,77,78]$. Tests of free volume models to experimental results revealed the general failure of such approaches [79, 80]. Nowadays, a number of experiments on the pressure/temperature dependence of the relaxation time of the $\alpha$ process have evidenced how this is only partly determined by its specific volume, and therefore the amount of free volume, the rest being governed by thermal activation [81, 82, 83, 84].

\subsection{Approaches based on the entropy}

A common point between the dynamic and thermodynamic description of glasses is the possible existence of a singularity at a finite temperature. In particular, the Kauzmann $\left(T_{K}\right)$ and the Vogel $\left(T_{0}\right)$ temperatures define the point of zero excess entropy $\left(S_{\text {glass }}=S_{\text {crystal }}\right)$ and divergence of the relaxation time respectively. Starting from these premises, Gibbs and DiMarzio (GB) [85] formulated a quasi-lattice theory on linear amorphous polymers predicting an Ehrenfest second order thermodynamic transition at $T_{K}$. At this temperature, an "ideal" glass with vanishing configurational entropy $\left(S_{c}\right)$, as previously noticed by Kauzmann [6], is formed. According to this theory a concomitant divergence of the relaxation time would occur at $T_{K}$. This aspect of the theory was developed in a subsequent work by Adam and Gibbs (AG) [86] relating the relaxation time $(\tau)$ to the $S_{c}$ :

$$
\tau=\tau_{0} \exp \frac{\Delta \mu s_{c}^{*}}{T S_{c}(T)}
$$

where $\Delta \mu$ and $s_{c}^{*}$ are the energy barrier per particle over which a relaxing unit must pass and the configurational entropy associated to such a relaxation.

An important aspect of the GD-AG theory is that the relaxation associated to glassy dynamics occurs via a cooperative rearrangement of several basic structural units. In polymers, this does not necessarily correspond to the monomer size [87, 88]. Furthermore, the GB-AG theory suggests that the size of the cooperative region $z^{*}$, i.e. the number of units rearranging cooperatively during the relaxation, increases with decreasing temperature and, importantly, such an increase is directly correlated to the decrease in the $S_{c}: z^{*} \sim S_{c}^{-1}$. Several approaches have been presented in the search for the cooperative length scale. Among them we mention the random first order transition (RFOT) theory [89], Donth's approach [90, 91, 92], those based on stringlike motion [93], the four points dynamic susceptibility [94], and the self-concentration [95]. However, the direct correspondence with the GB-AG length is questionable and matter of open debate $[96,97]$.

Examination of eq. 6 indicates divergence of $\tau$ at the temperature where the $S_{c}$ drops to zero. Application to experimental data [98, 99] and simulations [100, 101, 96], where $S_{c}$ was employed as in the original formulation of the GD-AG theory, turned 
out to be generally successful. However it is worth mentioning that, when the entropy theory was formulated, the $S_{c}$ was identified with the entropy of the glass former in excess to that of the crystal $\left(S_{e x}\right)$. The latter was previously the object of Kauzmann analysis [6]. Serious criticism to the connection between the Gibbs-DiMarzio theory and Kauzmann experimental observations was subsequently raised by Goldstein and co-workers $[102,103]$ and more recently by Johari [104]. They noticed that the entropy of a supercooled liquid in excess to that of the corresponding crystal exhibits significant contribution from the excess vibrational entropy and, therefore, $S_{e x}$ and $S_{c}$ cannot be identified. Further criticism was put forward by Dudowicz et al. [105], who emphasized the substantial deviation at temperatures substantially above $T_{g}$ of the $S_{c}$ derived from relaxation time data via the $\mathrm{AG}$ equation and the $S_{e x}$ obtained from calorimetric data.

Notwithstanding the mentioned arguments of criticism, tests of the AG equation to experiments employing $S_{e x}$ in a relatively wide temperature range above $T_{g}$ [106], including those on miscible polymer blends [52, 107, 108] and mixtures of low molecular weight glass formers [109], and those performed under hydrostatic pressure $[110,111,112]$, in polymerizing glass formers [113], and metallic glasses [114] have proved to be successful. The reason for the validity of the AG equation with $S_{e x}$ likely lays in the proportionality of this magnitude with the $S_{c}$, at least in a temperature range relatively close to $T_{g}$. In this sense analysis of NS data on glassy selenium (Se) [115] allowed extracting the vibrational contribution to $S_{e x}$. This analysis showed that $S_{e x}$ $S_{c}$ are actually proportional over a relatively large temperature range [116]. Additional indications of such proportionality were provided by other authors employing different approaches [110, 117, 118].

\subsection{Thermodynamic description of the kinetic fragility}

The ability of the AG equation with the $S_{e x}$ to fit experimental data indicates that divergence of the relaxation time would occur at $T_{K}$. This suggests that $T_{K}$ and $T_{0}$ of the VFT equation should be equal. This has been tested for a large variety of glass forming liquids exhibiting $T_{K} \approx T_{0}$ [119]. A further step forward in the connection between dynamics and thermodynamics was performed when the kinetic fragility was related to the temperature dependence of the $S_{e x}$. This has been done by Angell and co-workers in several studies $[120,121,122]$. In Refs. [120, 121] the kinetic fragility was defined as the temperature at which the relaxation time on a log scale is halfway (or $3 / 4$ the way) between its value at $T_{g}\left(\tau \approx 10^{2} \mathrm{~s}\right)$ and the value at infinite temperature $\left(\tau \approx 10^{-14} \mathrm{~s}\right)$ and the thermodynamic fragility as that at which half the entropy of fusion has been lost on cooling. Conversely in Refs. [122] the steepness index ( $m$ from eq. 3) is related to the specific heat jump at the $T_{g}$ and the enthalpy of fusion $\left(\Delta S_{m}\right)$ via the equation: $m=40 \Delta C_{p} / \Delta S_{m}$, derived from the RFOT of Wolynes and co-workers [89]. The latter constitutes one of the most acknowledged among thermodynamic entropybased theories of the glass transition.

These results generally suggest that the the thermodynamic approach via the $S_{e x}$ 
to the dynamics of glass-forming liquids can be considered valid. At the same time it has to be noticed that the vast majority of the tests based on such approach are limited to a temperature interval above $T_{g}$. This is due to the fact that at lower temperatures the slowing down of the dynamics imposes long observation time scales. The main implication of the scarcity of data in the sub- $T_{g}$ regime is that the fate of both dynamics and thermodynamics close to $T_{K}$ and $T_{0}$ remains unknown and subject to speculations based on extrapolations. This point will be addressed in a subsequent section (Section 5) of the present Review.

\subsection{Specificity of polymers and other complex glass formers}

In the previous subsection, the ability of the GD-AG based thermodynamic approach to the dynamics has been reviewed. In particular, a number of studies where this approach was shown to be successful has been recalled. The situation becomes somewhat more complicated once polymeric glass formers are considered. With regard to the fragility, the relation between the kinetic and thermodynamic ones, originally developed by Angell and co-workers $[120,121,122]$, was questioned by several authors when analysing data on polymeric glass formers. Ngai and Yamamuro [123] and Roland and co-workers $[124,125]$, after defining the thermodynamic fragility as equal to the jump of the specific heat at the glass transition temperature $\left(T_{g}\right)$, found no correlation of this magnitude with the kinetic fragility, this expressed in terms of the steepness index $m$. Their definition of the thermodynamic fragility relies on the empirical idea that the rapidity of change of $\tau$ with temperature must be correlated to that of thermodynamics properties, a fact that would be caught by the difference between the melt and the glass heat capacity at $T_{g}$. As an example, Fig. 5 shows the kinetic fragility as a function of the specific heat jump at $T_{g}$ for series of amorphous PS and poly(dimethyl siloxane) (PDMS) with varying molecular weight [124]. As can be observed, the expected increase of fragility with the specific heat jump at $T_{g}$ is not encountered for these polymers. A similar analysis was conducted by McKenna [126], who neither found a straightforward correlation between kinetic and thermodynamic fragilities. In this case, the definition of the thermodynamic fragility was based on the ratio of the melt to glass heat capacity.

At the time of the introduction of the mentioned studies into the scientific debate, it was recognized that the origin of the failure of thermodynamic entropy based approaches to the fragility was due to the polymeric nature of those glass formers for which those approaches did not work. However, a first step toward the clarification of the criticism raised was provided by Mohanty et al. [127], who, rather than defining the thermodynamic fragility on an empirical basis, obtained it by simply introducing the AG equation into the expression of the fragility (eq. 3). In such a way the thermodynamic fragility was derived:

$$
m=\frac{\Delta \mu s_{c}^{*}}{\ln (10) T_{g} S_{e x}\left(T_{g}\right)}\left(1+\frac{\Delta C_{p}\left(T_{g}\right)}{S_{e x}\left(T_{g}\right)}\right)
$$




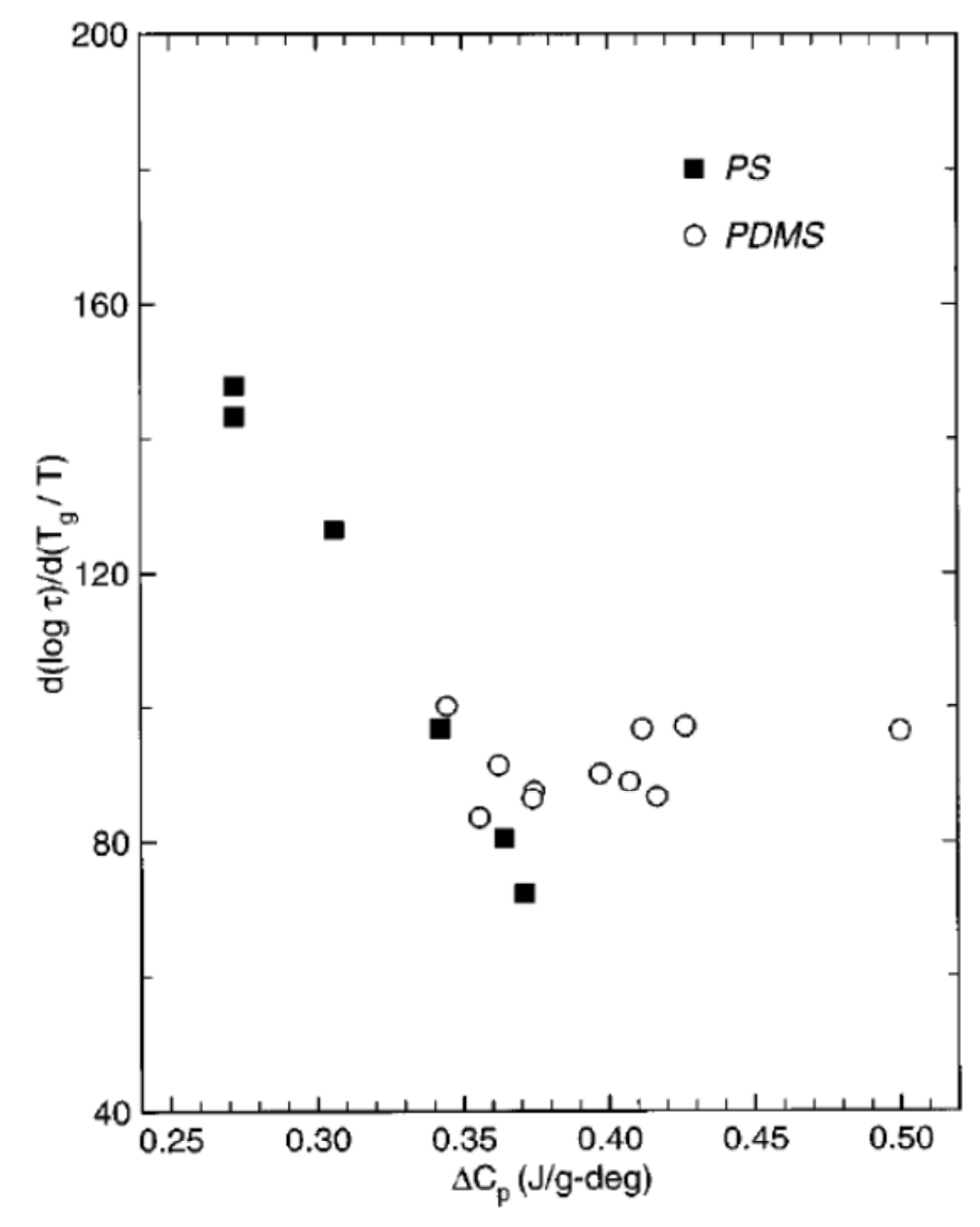

Figure 5. Kinetic fragility expressed in terms of the steepness index (see Eq. 3) as a function of the specific heat jump at $T_{g}$ for PS and PDMS with different molecular weights. (reprinted from ref. [124]).

Similar expressions for the thermodynamic fragility, all bearing a strong connection to the AG equation [86], have been subsequently derived [128, 129].

For a class of polymeric glass formers the relation kinetic versus the AG based thermodynamic fragilities via eq. 7 is found to hold [129]. However, despite the introduction of this new way of defining the thermodynamic fragility, still the connection with the kinetic fragility for a significant number of glass-forming polymers was not fulfilled. This has been noticed by Cangialosi et al. [129]. In the same work as well as in a previous one [130], it was contextually noticed that - for those polymeric glass formers not verifying the relation kinetic/thermodynamic fragility - the Vogel temperature was 
systematically larger than the Kauzmann temperature $\left(T_{0}>T_{K}\right)$. In the same context, for the same glass-forming polymers it was shown that the AG equation was apparently unable to catch the slowing down of glassy dynamics with temperature reduction [130].

The possible origin of the inability of the AG based thermodynamic approach to the dynamics could be sought in the previously discussed issue of the inequality between $S_{e x}$ and $S_{c}$. Furthemore, this inequality has been observed not only in polymeric glasses but also in several multicomponent bulk metallic glass forming systems [99]. Regarding polymers, it has be to remarked that the low energy density of vibrational states, from which the vibrational part of $S_{e x}$ originates, seems to be rather similar for all polymers. To account for the apparent inability of the thermodynamic approach, based on the AG equation, to provide a description to the fragility in a considerable number of polymeric glass formers, an aspect that was emphasized was that such polymers exhibit prominent secondary relaxation processes $[129,130]$. These are inevitably associated to a number of degrees of freedom, which contribute to the $S_{e x}$, since such processes are not present in crystals. In this context, the main challenge is the derivation of such contribution. This could be in principle done if the number of degrees of freedom of secondary relaxations was exactly known. However the following approximation can be reasonably made to extract the contribution to the $S_{e x}$ from secondary relaxations $[129,130]$ : i) in view of the absence of any contribution to $S_{e x}$ from the $\alpha$ process at the Vogel temperature alleged by the GB-AG theory, the residual $S_{e x}$ at such temperature is exclusively related to secondary relaxational processes, that is, $S_{e x-s e c} \approx S_{e x}\left(T_{0}\right)$; ii) considering that secondary relaxations generally do not contribute to the specific heat of glass forming materials [131, 132] (no jump in specific heat is observed, when the experimental time scale of a DSC experiment is of the order of that of secondary relaxations [40]), the $S_{e x}$ from these processes is approximately temperature independent. The latter observation implies that at $T_{g}$ (at which the fragility is defined) the $S_{e x}$ of secondary relaxations is nearly identical to that at the Vogel temperature: $S_{e x-s e c} \approx S_{e x}\left(T_{0}\right) \approx S_{e x-s e c}\left(T_{g}\right)$. This provides a correction for the thermodynamic fragility in eq. 7 , where the total $S_{e x}$ is replaced by: $S_{e x-\alpha}\left(T_{g}\right)=S_{e x}\left(T_{g}\right)-S_{e x-s e c}$. Fig. 6 shows the connection kinetic versus thermodynamic fragilities for several glass forming polymers before and after the correction accounting for secondary relaxations. Here, both the kinetic and thermodynamic fragilities are defined following Speedy [133]. These can be rescaled to those usually employed in the literature for the steepness index [21] by simply multiplying by a factor (decimal) $\log \tau_{0}+2$ (s).

The review of the developments in the field of thermodynamic approaches to the fragility reveals that the theoretical expectations of entropy based theories such as the GD-AG [85, 86] and RFOT [89] are fulfilled once a careful analysis is carried out. In particular, it must be taken into account that the kinetic fragility is defined exclusively for the $\alpha$ process. This implies that, whatever the metrics employed to define the thermodynamic fragility, this must contain only the contribution of those degrees of freedom associated to such relaxational process. This provides a correct estimation of the thermodynamic fragility. 


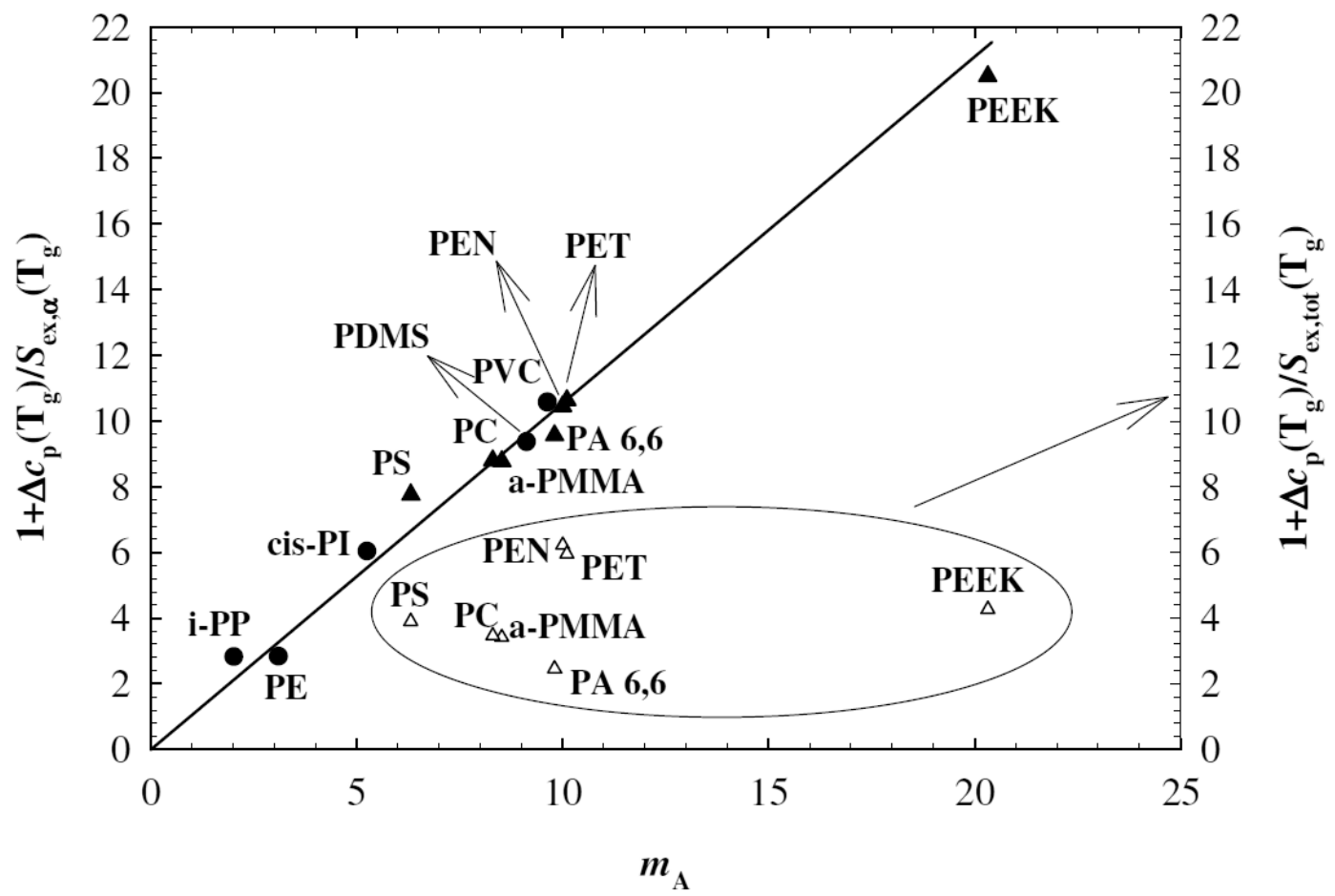

Figure 6. Thermodynamic fragility as obtained from the AG equation as a function of the kinetic fragility defined according to Ref. [133]: (right axis and open symbols) uncorrected; and (left axis and full symbols) corrected accounting for the contribution associated to secondary relaxation processes (reprint with permission from Ref. [129]).

\subsection{Thermodynamic versus vibrational approach to the dynamics of polymeric glass} formers

In the preceding section, the main aspects of the thermodynamic approach to the dynamics of glass-forming systems, with special attention to glass forming polymers, have been reviewed. However, the description of the kinetic fragility has been tackled employing a number of different approaches. Among them we recall the following ones: i) that based on the ratio of the between the maximum and the minimum of the boson peak, i.e., of the contribution observed at $\mathrm{THz}$ frequencies by Raman spectroscopy and neutron scattering [134]; ii) elastic approaches [75]; iii) the connection with the degree of stretching of the KWW function via the $\beta$ exponent [22]; iv) the approach based on the Poisson's ratio [135]; v) the connection with the the mean squared displacement (MSD) [136, 47]; iv) that with the low temperature vibrational properties [48].

Among the mentioned approaches, in at least two cases the question of the compatibility with the fragility of polymeric glass formers has been under query. These are that of the Poisson's ratio [135] and that based on the low temperature vibrational properties [48]. In the former case, a proportionality between the ratio of instantaneous bulk to shear modulus in the glassy state was found for a considerable number of non- 
polymeric glass formers [135]. However, once glass forming polymers are investigated within this framework, as for the thermodynamic approach, the situation becomes more complicated. In particular, is has been shown that that the approach based on the Poisson's ratio is suitable for low molecular weight PS, whereas it fails for PS with $M_{w}>500 \mathrm{~g} \mathrm{~mol}^{-1}$. In particular, the kinetic fragility of high molecular weight PS(s) are far too large to be correlated to the relatively small ratios of the instantaneous moduli. A similar scenario was found for polymerizing glasses [137].

In the case of the approach based on low temperature vibrational properties, Scopigno et al. [48] collected data on the fragility and the nonergodicity factor (NEF), that is, the long time plateau of the density autocorrelation function, for a considerable number of non-polymeric glass former. The latter was determined by measuring the elastic to inelastic scattering ratio in Inelastic X-ray Scattering (IXS) experiments. They showed how glass formers exhibiting relatively large density decorrelation at low temperatures are the highly fragile ones. Hence a linear correlation between the fragility and a parameter $\left(\alpha_{N E F}\right)$ related to the NEF was found. This is related to the NEF $(f(T))$ by: $f(T)=1 /\left(1+\alpha_{N E F} T / T_{g}\right)$. The correlation fragility versus $\alpha_{N E F}$ was later addressed by Buchenau and Wischnewski [138] considering compressibility and sound velocity data, from which $\alpha_{N E F}$ can also be derived. They showed that several glass-forming polymers do not follow the linear relation between $\alpha_{N E F}$ and $m$ (see Fig. 7). In particular, according to Buchenau and Wischnewski's analysis, those glassforming polymers deviating from the expected behaviour exhibit large fragilities and comparatively small $\alpha_{N E F}$ parameters.

Inspired by the correction to the thermodynamic fragility based on the contribution of secondary relaxations [130, 129], Scopigno et al. [139] pointed out that estimates of the NEF based on scattering ratio in IXS experiments, as well as from compressibility and sound velocity data, exclusively account for the decorrelation of density fluctuation due to vibrational degrees of freedom. Since the NEF is the long time plateau of the density autocorrelation function, the IXS and other methods can only be applied in its original implementation [48] when the only relaxation is that of the $\alpha$ process. In presence of secondary relaxations, faster than the structural process, intermediate additional plateaus will appear in the density autocorrelation, and a meaningful connection with the fragility of the glass former should rather isolate the contribution from the $\alpha$ relaxation only. In the work of Scopigno et al. [139] the degree of decorrelation resulting from secondary relaxations in the form of additional contributions in the elastic spectral response, is straightforwardly quantified based on the contribution of secondary relaxations to $S_{e x}$. In doing so, the correlation between $\alpha_{N E F}$ and the steepness index, $m$, is recovered for those glass forming polymers exhibiting significant decorrelation from secondary relaxation processes. This is shown in Fig. 8 where the kinetic fragility is plotted as a function of the corrected $\alpha_{N E F}[139]$.

All together, these findings demonstrated two important points in the description of the fragility of glass-forming liquids: i) the essential equivalence between the thermodynamic approach and that based on fast vibrational degrees of freedom; and ii) 


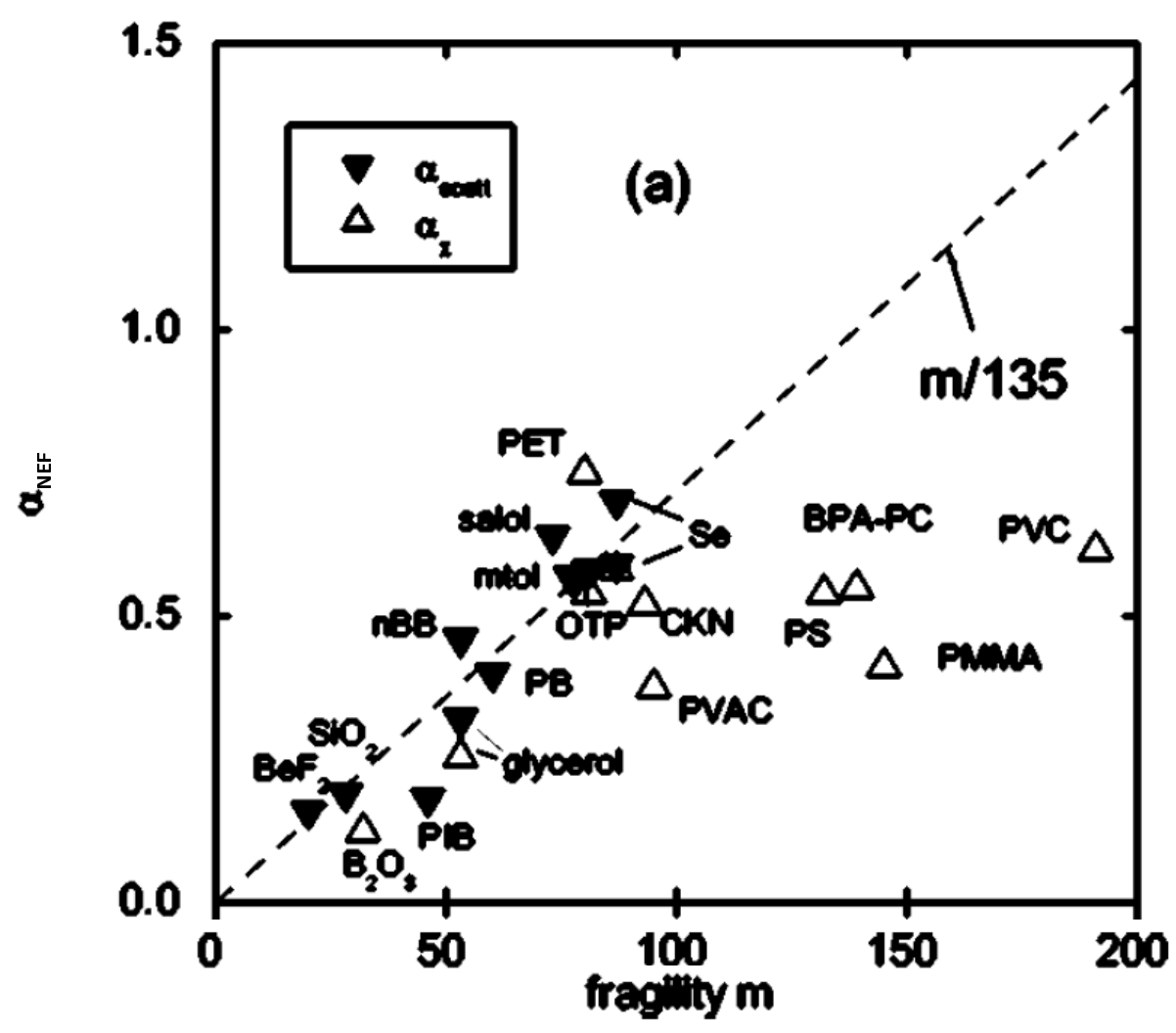

Figure 7. $\alpha_{N E F}$ parameter obtained from compressibility (open symbols) and sound velocity (closed symbols) data as a function of the steepness index (reprinted from Ref. $[138])$.

the peculiarity of polymeric glass formers due to the prominent contribution of secondary relaxations to both thermodynamic properties $S_{e x}$ and the density autocorrelation function.

Several studies have been recently presented reporting apparent outliers of either the thermodynamic or the vibrational approach to determine the fragility, even in nonpolymeric glass formers. Niss et al. [140] presented a detailed experimental study on the short wave-length properties probed by IXS on a significant number of glass formers. They showed that decahydroisoquinoline (DHIQ), a glass former with exceptionally high fragility $(m=155)$, exhibits strong deviations from the expected relation with the $\alpha_{N E F}$ parameter. However, DHIQ exhibits a strong secondary relaxation process, as shown by BDS [141], likely related to the considerable number of intra-molecular degrees of freedom possessed by this glass former. Hence, before drawing any final conclusions, the contribution of secondary relaxations to $\alpha_{N E F}$ parameter should be properly accounted for. Ruta et al. presented IXS results on glassy sorbitol [142]. The obtained $\alpha_{N E F}$ parameter was shown to be too small to be related to the fragility in the way proposed by Scopigno et al. [48]. As in the case of DHIQ, the apparent lack of correlation between the $\alpha_{N E F}$ parameter is likely to be sought in the presence of a strong secondary relaxation, intramolecular in nature, as detected by standard spectroscopic techniques such as BDS 


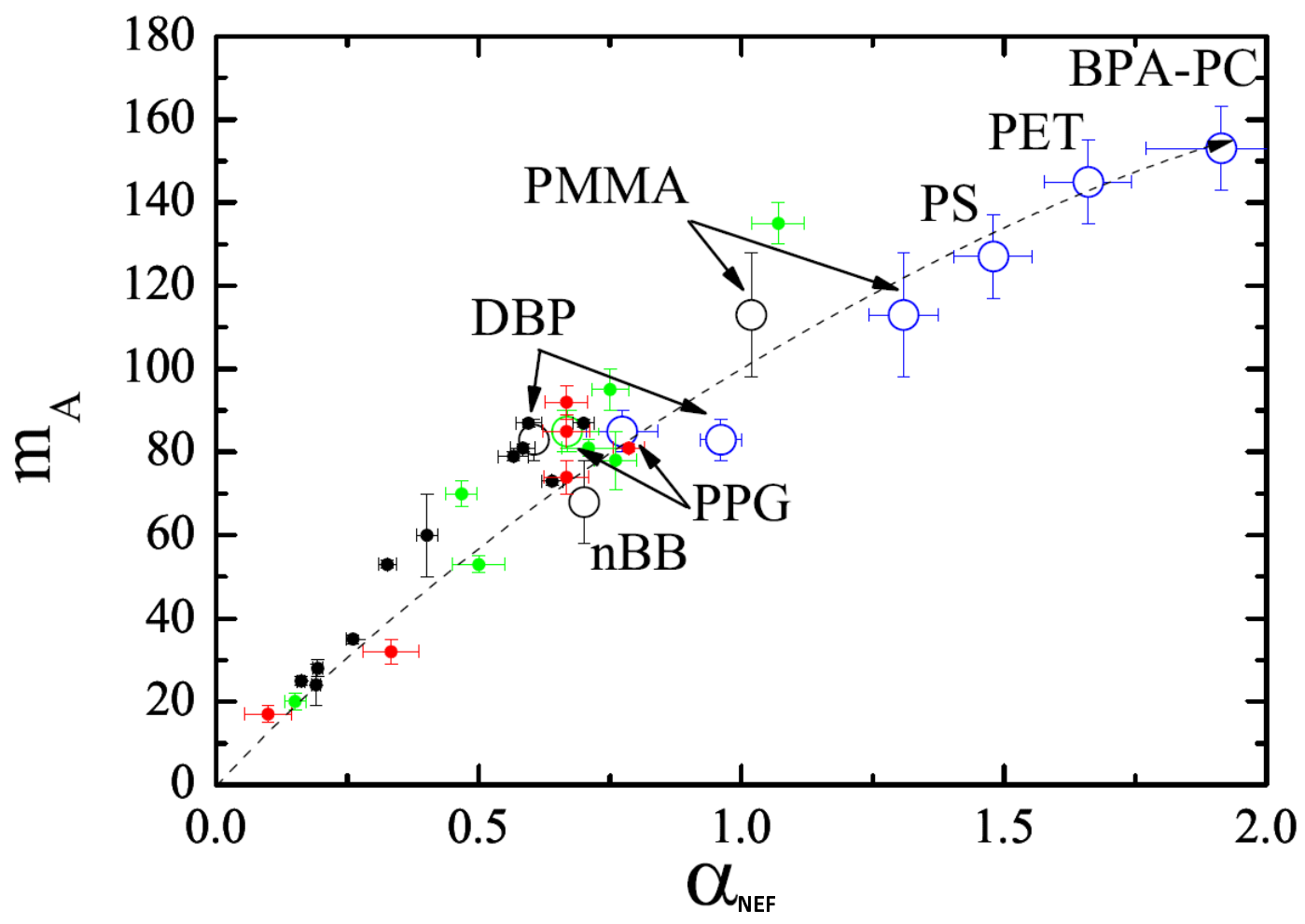

Figure 8. Steepness index as a function of the $\alpha_{N E F}$ parameter from the NEF obtained after accounting for the contribution of secondary relaxation processes (reprinted with permission from Ref. [139]).

[143]. Dalle-Ferrier et al. [144] investigated the molecular weight dependence of the correlation $\alpha_{N E F}$ parameter in polystyrene (PS) and polyisobutylene (PIB). They found that the correlation fragility versus $\alpha_{N E F}$ is lost once PS molecular weight is increased and that of PIB decreased. In the former case, it is interesting to recall a recent study on the dependence on the molecular weight of PS molecular dynamics [145]. This shows that high molecular weight PS exhibits pronounced secondary relaxation, whereas such process tends to disappear when lowering the molecular weight. At molecular weights smaller than $700 \mathrm{~kg} \mathrm{~mol}^{-1}$ no secondary relaxations can be detected. This result is a further strong indication of the need to properly determine the NEF in high molecular weight PS(s) for a meaningful comparison with the fragility. In the case of PIB, an opposite scenario exists: low molecular weight PIB exhibits significant deviations from the expected correlation of $m$ and $\alpha_{N E F}$. Although some studies reported the BDS response of PIB with some specific molecular weights [146, 147, 148], unfortunately systematic studies on the relaxation behavior of PIB as a function of the molecular weight have not been performed in the past. In particular, the presence of secondary relaxations in this polymer and whether such relaxations exist over the whole range of molecular weights should be explored for a correct interpretation of $m$ versus $\alpha_{N E F}$ results. 
In closing this section, we remark that the arguments raised for the correct determination of the thermodynamic fragility are valid whatever the approach followed for the description of the fragility. In particular, a detailed analysis of the global properties of the glass former under examination should be pursued. A straightforward comparison between properties representative of the overall nature of the glass formers and fragility, under the strong assumption of a single process, the structural relaxation, is not always possible. In this section the effect of secondary relaxations to the NEF has been emphasized.

\section{Dynamics and thermodynamics below $T_{g}$}

The previous sections of the Review have highlighted the suitability of the AG based approach to describe glassy dynamics. In particular the attention was devoted to tests of the relation between dynamics and thermodynamics above $T_{g}$. As a result, the presence of a second order thermodynamic transition at $T_{K}$ with divergence of the relaxation time cannot be unequivocally proved. In other words, one could put forward that deviations of both dynamics and thermodynamics from the expected behaviour occur somewhat below the $T_{g}$. In this section we discuss the recent activity devoted to unveiling the fate of the dynamics and thermodynamics toward the purported singularity at a finite temperature $\left(T_{K}\right.$ or $\left.T_{0}\right)$ below $T_{g}$.

\subsection{Dynamic aspects}

The experimental and theoretical activity aiming to get insight on the behaviour of glassy dynamics is currently a topic of intense investigation and open debate. Regarding experimental studies, it has to be remarked that studies of the dynamics in the glassy state are numerous. However, the vast majority of them concerns out-of-equilibrium glasses, that is, in the scheme of Fig. 1 glasses high in the energy landscape, that is exploring thermodynamic states with configurations with higher energy than those of the metastable equilibrium ones. The information obtained in this regime does not allow drawing any conclusion on the fate of the dynamics below $T_{g}$. This applies for numerous studies where the relaxation time is determined below $T_{g}$ in not-fully equilibrated samples [149, 150, 151, 152, 153, 154, 155]. Hence only those studies providing insight on the dynamics of equilibrated glasses will be considered, or, at least, those where it is not clear whether the glass is at the metastable equilibrium or not. In the latter cases, to avoid ambiguities, it will be explicitly mentioned.

5.1.1. Experimental studies The first experimental study addressing the question of whether the relaxation time follows the VFT behaviour below $T_{g}$ is that of Nozaki and Mashimo [156]. They employed BDS to investigate PVAc glassy dynamics at time scales as large as $10^{6} \mathrm{~s}$, that is in the sub- $T_{g}$ regime. The relaxation time exhibited clear deviations from the behaviour expected according to the VFT (or equivalently the WLF) 
equation. However, it is not clear whether, in the study of Nozaki and Mashimo [156], the obtained relaxation time corresponds to that of equilibrium, since no information on the annealing protocol below $T_{g}$ is provided in that study [156].

However, deviations from the VFT behaviour of the relaxation time are observed in equilibrated glasses or, at least, in glasses where no apparent evolution of the thermodynamic state is observed. The first of such studies was presented by O'Connell and McKenna [157] for polycarbonate (PC). They showed that the shift factor obtained from time-aging time superposition in stress relaxation experiments reached a plateau by extended annealing below $T_{g}$. When the limiting shift factor was plotted as a function of temperature, clear deviations from VFT behaviour toward milder Arrhenius temperature dependence were found. McKenna and co-workers later extended such studies to other systems, in particular PVAc, for which analogous deviations were detected [158]. Indications on the non-diverging scenario, that is, deviating from the VFT law, were provided by Simon et al. [70] investigating the kinetic of enthalpy and volume recovery of glassy PS. Arrhenius temperature dependence was found once the shift factor obtained by time-aging time superposition of enthalpy recovery data close to plateau was derived. A similar investigation was conducted by Boucher et al. [71] on several glass forming polymers. The shift factor obtained from superposition of enthalpy recovery data close to the plateau was found to be much smaller than predicted by the VFT equation. Deviations from VFT behaviour were found by Thurau and Ediger exploring the fate of the segmental relaxation below $T_{g}$ in PS and PC following the diffusion of probe molecules by an optical photobleaching technique [25, 159]. A model dependent analysis of the free volume evolution, probed by PALS during physical aging of PC, indicates a crossover of the relaxation time from VFT to Arrhenius behaviour, the latter with activation energy considerably lower than that of the $\alpha$ process around $T_{g}$ [160]. A very recent study on 20 million years amber, where the dynamics is probed as a function of temperature by stress relaxation experiments, indicates that the instantaneous relaxation time dramatically deviates from the behaviour expected within the diverging scenario [161]. This is shown in Fig. 9 where the scaled relaxation time is reported as a function of the temperature together with the VFT expectation according to high temperature relaxational data.

With regard to non-polymeric glass formers, Kruger et al. [162] measured the time evolution during physical aging of the sound frequency, as obtained by Brillouin spectroscopy, and found deviations from VFT behaviour below $T_{g}$. However, in this case criticism has been raised concerning the actual measurement of equilibrium relaxation times [163]. Ruta et al. [68, 69] measured the evolution of the relaxation time during physical of metallic glasses well below their $T_{g}$ by XPCS. Despite the considerable distance from $T_{g}$ a plateau in the relaxation time is found after aging for days and, importantly, its limiting value appears to be far too small to be compatible with a VFT extrapolation. Welch et al. [164] measured the volume recovery of an aluminosilicate glass at $T \sim T_{g}-600 \mathrm{~K}$. As in the case of the works of Ruta et al. [68, 69] a plateau in the recovery was found in a relatively short time (less than one year). In the work 


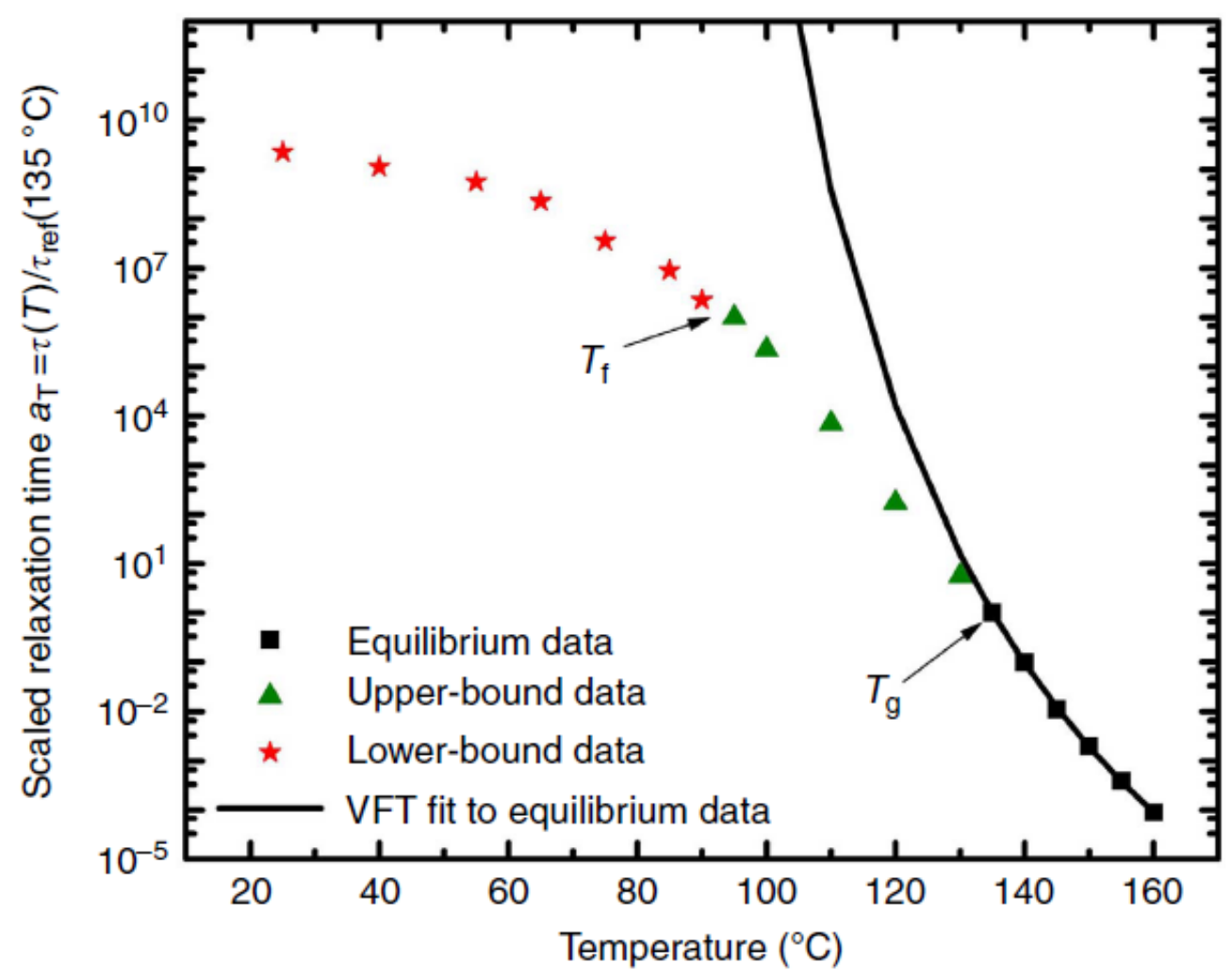

Figure 9. Temperature dependence of the scaled relaxation time of 20 million years aged amber, obtained from time-temperature superposition of stress relaxation curves (reprinted with permission from Ref. [161]).

of Welch et al. [164], the time scale of equilibration, once plotted as a function of the inverse temperature, significantly deviates from the VFT behaviour. Mismatches between the trend of the relaxation time in comparison to the prediction of the VFT law were reported by several other studies on low molecular weight glass formers $[165,166]$.

Beside the mentioned studies reporting deviation from the VFT behaviour, there exists a considerable number showing opposite trends. Alegria et al. [167] probed the aging time evolution of the $\alpha$ relaxation time on several glass forming polymers. Once the plateau in such evolution is achieved, the relaxation time follows the VFT equation. Richert and co-workers [168, 169] extended this kind of studies to temperatures even lower than those of Alegria et al. study [167]. In particular, they measured equilibrium relaxation times up to $10^{6} \mathrm{~s}$ of PVAc by BDS after aging for more than one year. In contrast with McKenna and co-workers finding [158], the equilibrium relaxation time followed the behaviour expected from the VFT equation. Though on shorter time scales, a similar result was found for the same polymer by Boucher et al. [170]. The apparent discrepancy between these results has very recently promoted the debate on the divergence versus non-divergent scenario of glassy dynamics [171, 172]. These contrasting results are summarized in a very recent work [171] and reported in Fig. 10. In this figure the scaled equilibrium relaxation times over a wide range of temperatures 
are showed, in particular below $T_{g}$. The different trend of the relaxation time found by Richert and co-workers $[168,169,171]$ on one side and McKenna and co-workers $[158,172]$ on the other will be discussed in detail in a subsequent section of the Review (Section 5.3).

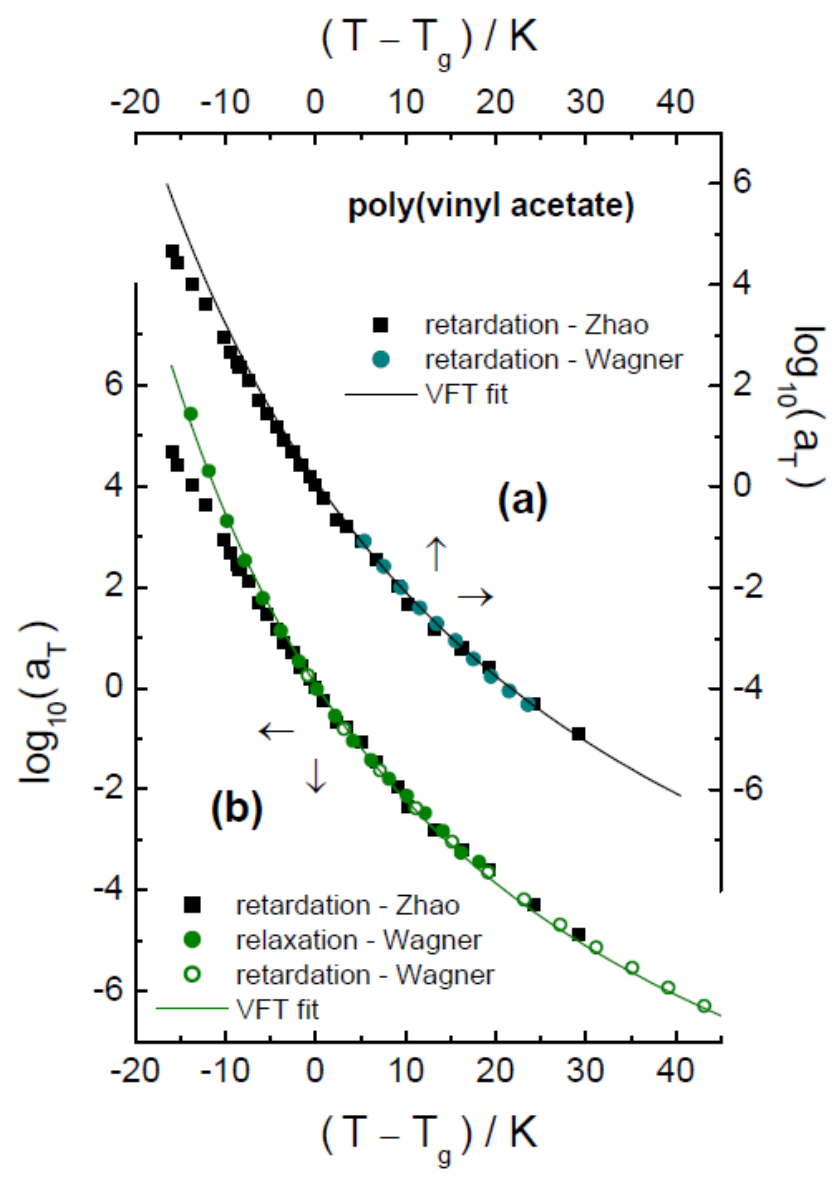

Figure 10. Scaled equilibrium relaxation time as a function of temperature for PVAc obtained by Wagner and Richert [168, 169] and Zhao and McKenna [158] (reprinted with permission from Ref. [171]).

To close this subsection, we briefly mention that a system for which the fate of the dynamics has been hotly debated in the last years is that of supercooled water. Due to its strong tendency of this system to crystallize, supercooled water has been investigated after cooling at high rates or in confinement. These methods, if appropriately employed, actually provide a route to avoid water crystallization. Some authors proposed that supercooled water undergoes a fragile to strong transition when cooling down [120] and some experiments have indicated the presence of such phenomenon [173]. In the context of the discussion of the present Review, this means that the rather pronounced temperature dependence of the relaxation time of supercooled water is not followed on cooling. In turn, this would either substantially reduce the Vogel temperature or completely avoid the singularity with diverging relaxation time at a finite temperature. 
The actual presence of the fragile to strong transition has been however criticised by several authors $[174,175,176,177,178,179]$. Nevertheless, the activity in this topic may in principle provide indications on universal features of the relaxational behaviour of glass forming materials, included polymers.

5.1.2. Theoretical approaches From a theoretical point of view, the GD-AG approach represents the first approach providing a prediction of the fate of the dynamics below $T_{g}$. The first theoretical approach questioning the idea of divergence of the relaxation time provided in the GD-AG theory was that of Avramov and Milchev [180]. Their atomic hopping model to glassy dynamics predicts non-divergent relaxation time. Subsequently a number of different approaches where introduced in the scientific debate providing a non-divergent description of the dynamics of glass forming liquids. Among them we recall: i) the description of DiMarzio and Yang [181] based on particle motion resulting from the escape from deep potential wells and swimming in a configurational sea; ii) the activated barrier hopping approach by Schweizer and co-workers $[182,183]$; iii) the kinetically constrained model delivering a quadratic (non-divergent) form of the relaxation time [184]; iv) the $S_{c}$ based approach by Mauro and co-workers $[185,186]$; v) the kinetic liquid model [187]; vi) the approach of Kivelson et al. [188], in which divergence of the relaxation time is avoided by geometric frustration. Furthermore, it has been shown that - once the deviation from VFT behaviour is incorporated in Kovacs-Aklonis-Hutchinson-Ramos (KAHR) model [189] for the description of physical aging - improved fits of volume recovery data on PVAc are achieved in comparison to those based on the description of the dynamics below $T_{g}$ via the VFT equation [190].

Beside models predicting deviations from VFT behaviour and beyond the GD-AG theory, different theoretical approaches predicting divergence of the relaxation time are of relevance. Among them the RFOT theory of Wolynes and co-workers [191, 192] and its development by Bouchaud and Biroli [193], the two-order-parameters (TOP) [194] and the replica theory [195] theory can be mentioned.

\subsection{Thermodynamic studies}

The vast majority of enthalpy or volume recovery studies mainly focuses on a temperature interval close to $T_{g}$. This is due to the relatively short time scales required for glasses to reach equilibrium. Alternatively a considerable number of studies is performed well below $T_{g}$ but during aging times not long enough to reach a plateau in the observable under consideration. A typical example of this kind of studies is that of volume recovery experiments by Kovacs [10]. Nevertheless, in recent years several studies pointed toward a non-trivial behaviour of thermodynamics significantly below $T_{g}$. In particular a number of works has been presented where either the enthalpy or the volume after prolonged aging reached a plateau with partial recovery, that is, where the thermodynamic state exhibits larger volume or enthalpy that those expected extrapolating from the melt state (see Fig. 1). This has been shown in several glass 
forming polymers including PC [196], PS [197, 198, 199], PVAc [200] styrene-acrylonitrile (SAN) copolymer [201] and poly(methyl methacrylate) (PMMA) [202]. Furthermore in a recent study [71], the plateau with partial enthalpy recovery is achieved by both upand down-jump experiments thus demonstrating that this corresponds to a (relative) minimum in the free energy. A notable outlier in this sense is a recent study where the enthalpy recovery is monitored during physical aging of PS at $358 \mathrm{~K}$ [203]. Here full enthalpy recovery is achieved in one decay despite the significant distance from PS $T_{g}$. Possible explanations to this result are discussed in a subsequent work [204].

In the context of the fate of thermodynamics below $T_{g}$, beyond those studies where the recovery of thermodynamic properties is investigated, other approaches have been employed to clarify the issue on whether the extrapolated line of the supercooled liquid is followed. A test of GD scenario with vanishing excess thermodynamic properties at the Kauzmann temperature have been pursued by Baschnagel et al. [205] by performing simulations within bond-fluctuation model, a lattice model for polymers. They showed that, in proximity of the Kauzmann temperature, the entropy deviates from the behaviour expected considering simulations data at higher temperatures. In such a way the singularity with vanishing $S_{e x}$ at a finite temperature is avoided. Considerable effort, analysing available calorimetric data, have been devoted by Johari [206, 207, 208]. According to his analysis, no vanishing $S_{e x}$ at a finite temperature should exist. From a theoretical point of view, Wunderlich [209] separated each contribution to the heat capacity (vibrational, configurational and external contributions) of polyethylene (PE). In doing so he found a positive residual entropy at $0 \mathrm{~K}$ at odds with the alleged singularity at the Kauzmann temperature.

\subsection{Complex behaviour of dynamics and thermodynamics below $T_{g}$}

So far the thermodynamic and dynamic description of glasses below $T_{g}$ was based on the assumption of a simple behaviour, that is, a single decay to the ultimate equilibrium of thermodynamic properties. Recently, several experimental studies have opened the possibility that recovery of equilibrium occurs in a more complex fashion. The first of this kind of study was presented in the mid '90ies by Wimberger-Friedl and DeBruin [210]. They studied the volume recovery of PC at room temperature, that is, well below the polymer's $T_{g}$, over aging times as large as several years. They found a nonmonotonic decay of the rate of volume recovery. In particular, such rate appears to increase suddenly as the aging time is of the order of $10^{7} \mathrm{~s}$. A very similar results was presented some years later by Wilkes [211] for PC. In the same study the volume recovery of PS was shown to exhibit no indication of a crossover in the aging rate in the investigated time window (up to almost $10^{8} \mathrm{~s}$ ).

A systematic study on the long term aging behaviour on different polymeric glass formers has been recently presented [54]. In this study the enthalpy recovery of PC and, low and high molecular weight PS was investigated for aging times larger than those of the studies reporting partial enthalpy recovery [196, 197, 198, 200, 201, 202, 71]. From 
the point of view of thermodynamics, it was found that, apart from the decay to the plateau with partial enthalpy recovery, a second decay occurred until complete recovery. This is shown in Fig. 11 (upper panel) for high molecular PS as a showcase. As can be observed, on lowering the aging temperature, the enthalpy recovery pattern splits in two decays and the separation between them increases.

In the same study [54], information regarding the time scale of equilibration was achieved. This is shown in the lower panel of Fig. 11. The two time scales corresponding to each equilibration plateau exhibit significantly different behaviour: i) Arrhenius temperature dependence is found for the shorter equilibration time scale with activation energy several time smaller than that of the $\alpha$ process; ii) the time scale of complete equilibration exhibits temperature dependence typical of the polymers' $\alpha$ process. The latter point is evidenced by the ability of the VFT equation - with $B$ and $T_{0}$ taken from independent measurements by BDS - to catch the temperature dependence of the ultimate equilibration time (continuous lines in the lower panel of Fig. 11).

The presence of multiple equilibration mechanisms constitutes an important novelty in the understanding of the thermodynamics and dynamics of polymer glasses. Interestingly, the presence of multiple plateaus in the enthalpy recovery has been recently observed in the enthalpy recovery of arsenide selenide glasses aged for up to 25 years [212] and in glassy glycerol monitoring the Brillouin frequency shift during physical aging [213]. These results insinuate universality of such behaviour. Furthermore, all those studies reporting partial recovery of thermodynamic properties [196, 197, 198, 199, 200, 201, 202] need to be reconsidered in view of the possible evolution of those properties at larger aging times.

Apart from those studies where multiple equilibration mechanisms have been unequivocally shown, it is worth of remark that numerous studies have shown the presence of multiple endothermic peaks in the specific heat versus temperature scans in both polymeric and non-polymeric glass formers [214, 215, 216, 217]. The endothermic overshoot, as shown in Fig. 2, is a manifestation of the recovery of the enthalpy state of the supercooled liquid. Such behaviour has been usually attributed to the nonlinear behaviour of physical aging [215]. This is due to the fact that, in the physical aging regime, the relaxation time evolves with the annealing time as a consequence of densification. As a matter of fact, one of the most employed model to describe the recovery of thermodynamic properties of glasses - that is the Tool-NarayanaswamyMoynihan (TNM) [218, 219] model, where non-linearity is contemplated - is able to account for the presence of multiple endothermic overshoots. However, in view of the multiple equilibration mechanisms found very recently [54], an alternative explanation can be provided to the presence of several endothermic overshoots in the specific heat when heating up a glass well aged below its $T_{g}$. Further investigation is required to clarify this point.

The complex behaviour of glass equilibration offers a new perspective for the comprehension of literature data. This concerns the recent debate on whether the dynamics of glassy PVAc follows VFT behaviour [168, 169, 171] or deviates from it 

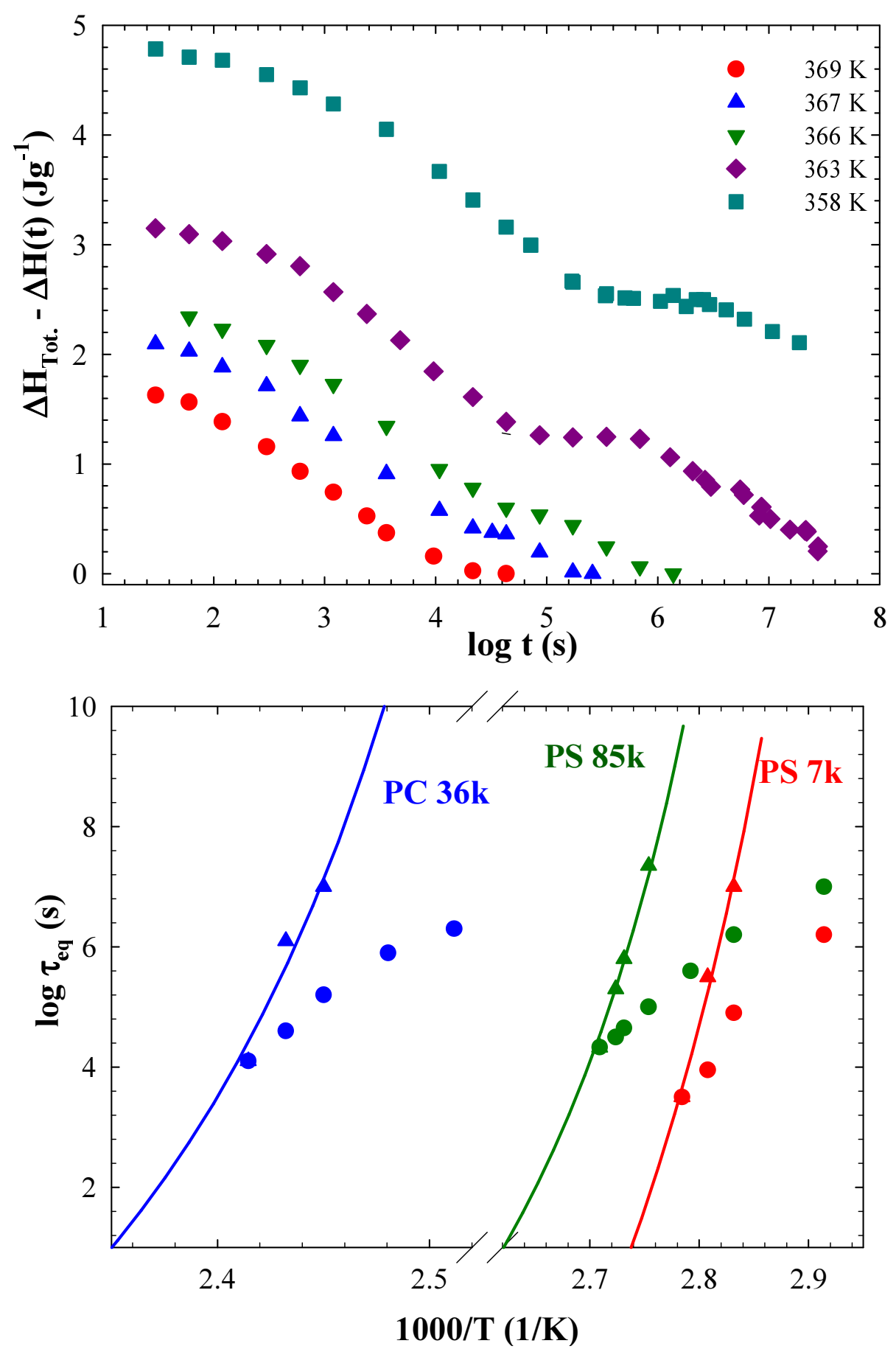

Figure 11. (Upper panel) Enthalpy recovery, expressed in term of the distance from equilibrium, as a function of the aging time at different temperature for high molecular weight PS. (Lower panel) Temperature dependence of the equilibration time for the first (circles) and second decay (triangles) in the enthalpy recovery of different polymers (reprinted from Ref. [54]).

$[158,172]$. In this sense, it is important to highlight the experimental conditions employed to achieve these apparently contradicting conclusions. In the studies by 
Richert and co-workers [168, 169], PVAc samples are aged during one year or more and only afterwards the relaxation time time is measured. Hence, rather than the evolution during equilibrium recovery, a single point, that is, the equilibrium relaxation time, is determined. Conversely McKenna and co-workers [158, 172] monitored the evolution of the relaxation time continuously over a time scale not larger than 20 days. In view of the results of Ref. [54], it is possible to speculate that the plateau in the relaxation time found after 20 days or less corresponds to partial recovery of thermodynamic properties, whereas the equilibrium relaxation time obtained after one year aging is the ultimate one. As an alternative explanation, it is worth mentioning that analysis of Richert and co-workers relaxational data based on time-temperature superposition, rather on fitting by the KWW equation, indicates deviations from the VFT behavior even for PVAc aged for more than 1 year [172]. These possible explanations can be verified by monitoring the evolution of the relaxation time over time scales longer than those employed in Refs. $[158,172]$.

The experimental finding of multiple equilibration steps needs to be described by suitable theoretical frameworks and this constitutes a challenge toward the understanding of the entire phenomenology of the glass transition. Very recently the application of the RFOT theory [220] to the out-of-equilibrium dynamics in the glassy state showed remarkably good agreement [221] with the two time scales found experimentally in PS [54]. Within this framework, a bimodal distribution, giving rise to the two steps decay, naturally originates from the evolution of dynamical heterogeneity of glassy dynamics. A different view to explain experimental facts, not necessarily in contradiction with that based on the RFOT theory, may originate from the inability of $\alpha$ process to entirely describe mechanical relaxation data close to and below $T_{g}$ [222].

\section{Summary and Conclusions}

In the previous sections the need for routes to achieve glasses low in the energy landscape has been emphasized in relation to the topic of the dynamics and thermodynamics in glass forming polymers and their possible connection. Aging a glass over extremely long times constitutes a possible way to achieve equilibrated glasses in the sub- $T_{g}$ regime $[210,211,212,54]$. An interesting route in this sense is that of employing specimens naturally aged over extremely long periods of time. This is the case of the study of McKenna and co-workers [161] on 20 millions years aged amber. However the limitation of this kind of studies is that the characterization of a glass very low in the energy landscape is possible for a single aging time and no information on how such a low energy state is achieved can be obtained.

Insight from low energy glasses can be obtained by physical vapour deposition. This consists in evaporating the glass forming material in high vacuum layer by layer on a substrate. It has been shown that this allows producing glasses with thermodynamic properties considerably lower in the energy landscape than those of conventional glasses, e.g. those produced by simple cooling through the $T_{g}$. In particular, it has been shown 
that, for several vapour deposited low molecular weight glass forming systems, the $T_{f}$, whose value is a measure of the location in the energy landscape of the glass, was much lower than that achievable by prolonged aging after cooling from above $T_{g}[223,224]$. Apart from the thermodynamic implications of this kind of study, interestingly it has been shown that some of the so-obtained glasses exhibit enhanced local orientational order [225], in agreement with theoretical expectations [194]. Furthermore, after measuring the local structure of vapour deposited glasses by wide angle X-ray scattering, it has been proposed that a new thermodynamic phase is formed by this procedure and, therefore, a first order thermodynamic transition restoring the conventional supercooled liquid would take place once the vapour deposited glass is heated well above its $T_{g}$ [226]. Hence, it remains to be clarified whether vapour deposited glasses are actually representative of systems aged after huge time scales or, alternatively, a glass bearing only the thermodynamic state of a well aged one is formed by this route. The latter scenario implies that glasses need to be described by several order parameters. These issues certainly are worth being explored in the future. Unfortunately, the method to obtain glasses low in the energy landscape based on physical vapour deposition is not applicable to polymers. However, it has to be mentioned that the employment of such method has recently promoted the scientific activity devoted to the search for new routes to obtain polymeric glasses with thermodynamic properties markedly different from those of glasses obtained by conventional routes [227].

In the search for a route to obtain low energy glasses, confinement of glass forming polymers may represent a suitable one. The effect on glassy dynamics of reducing one or more dimensions has been deeply explored in recent years with particular reference to the variation of the $T_{g}$ [228]. Among the different geometrical configurations that of thin polymers films, that is, films with thickness below about $100 \mathrm{~nm}$, represent by far the most investigated since the work of Keddie and Jones [229]. They measured the thickness of thin PS films supported on silica by ellipsometry and found a reduction in $T_{g}$. Since then numerous studies have been presented. Similarly to the work of Keddie and Jones, most of them report $T_{g}$ reduction. Several studies provide a comprehensive review of the rich literature dealing with the $T_{g}$ of thin polymers films [230, 231, 232]. Within the context of the present work, what is important to remark is that, until a certain extent, the $T_{g}$ reduction in confinement does not imply a concomitant speed-up in the polymer molecular mobility [62, 233, 234, 235]. This has been unequivocally shown in thin PS films [236] as well as PS nanospheres [237]. Furthermore, it has been shown that the thermodynamics of freestanding thin polymer films is sensitive to the thickness only below values [238] smaller than those for which effects on the $T_{g}[239,240,241,242,243,244,245,234,246,236]$ and the physical aging behaviour $[247,248]$ are observed. In particular, effects on the thermodynamics of freestanding thin PS films are observed below $30 \mathrm{~nm}$, whereas, for the confining configuration, $T_{g}$ reduction and alteration of the physical aging behaviour are observed for films as thick as $100 \mathrm{~nm}$ or more. Hence, the lower $T_{g}$ and accelerated physical aging found in confinement should not be attributed to the different dynamics and thermodynamics of the supercooled melt 
in comparison to the bulk counterpart. These results are simply an indication of the more efficient ability of maintaining equilibrium of glass forming systems under certain conditions of confinement. From the point of view of the topics reviewed in the present work, this implies that the dynamics and thermodynamics of glasses can be explored low in the energy landscape simply employing confined systems bearing identical properties as those of the bulk glass except for the mentioned efficiency in maintaining equilibrium. Interestingly, a recent study on temperature dependence of the thickness of thin PS films by ellipsometry provides evidence for the presence of two jumps in the thermodynamic coefficient, in this case the linear coefficient of thermal expansion, at observation time scales (related to the inverse of the employed cooling rate) of the order of seconds [245]. This suggests that two equilibration time scales exist in thin PS films, a result analogous to that found in bulk by monitoring the enthalpy recovery of several polymer glasses in the physical aging regime [54]. Here the only difference is that the more efficient equilibration of thin films allows probing the presence of two mechanisms of equilibration over significantly shorter time scales (seconds [245] as compared to years [54]). Furthermore, the mentioned characteristic of confined glasses allows accessing the relaxational behaviour at equilibrium well below the bulk $T_{g}$. Several studies point toward a somewhat complex behaviour with rich relaxational pattern in equilibrated thin polymer films low in the energy landscape [232, 249, 250]. These aspects are certainly of interest and may provide new impetus for future work.

\subsection{Acknowledgments}

The authors acknowledge the University of the Basque Country and Basque Country Government (Ref. No. IT-654-13 (GV)), Depto. Educación, Universidades e investigación; and Spanish Government (Grant No. MAT2012-31088) for their financial support.

\section{Bibliography}

[1] Debenedetti P G 1996 Metastable Liquids: Concepts and Principles (Princeton University Press, Princeton)

[2] Schmelzer J W P, Gutzow I S, Mazurin O V, Priven A I, Todorova S V and Petrov B P 2011 Glasses and the Glass Transition (Wiley-VCH Verlag GmbH Co. KGaA, Weinheim)

[3] Perez J 1998 Physics and Mechanics of Amorphous Polymers (A. A. Balkema, Rotterdam)

[4] Strobl G 2007 The Physics of Polymers (Springer, Berlin-Heidelberg-New York)

[5] Prigogine I and Defay R 1954 Chemical Thermodynamics (Longman, London)

[6] Kauzmann W 1948 Chem. Rev. 43 219-256

[7] Angell C 1995 Science 267 1924-1935

[8] Debenedetti P G and Stillinger F H 2001 Nature 410 259-267

[9] Simon F 1931 Z. Anorg. Allg. Chem. 203 219-227

[10] Kovacs A J 1963 Fortsch. Hochpolym. Fo. 3 394-508

[11] Struik L C E 1978 Physical Aging in Amorphous Glassy Polymers and Other Materials 1st ed (Amsterdam, The Netherlands: Elsevier Science)

[12] Hutchinson J M 1995 Prog. Pol. Sci. 20 703-760 
[13] McKenna G B 2012 Physical Aging in Glasses and Composites (Springer US, New York, NY)

[14] Cangialosi D, Boucher V M, Alegría A and Colmenero J 2013 Soft Matter 9 8619-8630

[15] Tool A 1946 J. Am. Ceram. Soc. 29 240-253

[16] Vogel H 1921 Phys. Z 22 645-646

[17] Fulcher G S 1925 J. Am. Ceram. Soc. 8 339-355

[18] Tammann G and Hesse W 1926 Z. Anorg. Allg. Chem. 156 245-257

[19] Stickel F, Fischer E W and Richert R 1996 J. Chem. Phys. 104 2043-2055

[20] Williams M L, Landel R F and Ferry J D 1955 J. Am. Chem. Soc. 77 3701-3707

[21] Angell C A 1991 J. Non-Cryst. Solids 131 13-31

[22] Böhmer R, Ngai K L, Angell C A and Plazek D J 1993 J. Chem. Phys. 99 4201-4209

[23] Williams G and Watts D C 1970 Trans. Farad. Soc. 6680

[24] Sillescu H 1999 J. Non-Cryst. Sol. 243 81-108

[25] Hwang Y, Inoue T, Wagner P A and Ediger M D 2000 J. Polym. Sci. Pt. B-Polym. Phys. 38 $68-79$

[26] Richert R 2002 J. Phys.: Condens. Matter 14 R703-R738

[27] Palmer R G, Stein D L, Abrahams E and Anderson P W 1984 Phys. Rev. Lett. 53(10) 958-961

[28] Arbe A, Colmenero J, Monkenbusch M and Richter D 1998 Phys. Rev. Lett. 81(3) 590-593

[29] Cangialosi D, Alegría A and Colmenero J 2009 J. Chem. Phys. 130124902

[30] Schmidt-Rohr K and Spiess H W 1991 Phys. Rev. Lett. 66(23) 3020-3023

[31] Böhmer R, Diezemann G, Hinze G and Rossler E 2001 Prog. Nucl. Magn. Res. Spect. 39 191-267

[32] Schiener B, Böhmer R, Loidl A and Chamberlin R V 1996 Science 274 752-754

[33] Richert R 1997 J. Phys. Chem . B 101 6323-6326

[34] Cicerone M T and Ediger M D 1996 J. Chem. Phys. 104 7210-7218

[35] Bernabei M, Moreno A J and Colmenero J 2008 Phys. Rev. Lett. 101255701

[36] Dyre J C 2007 J. Phys.: Condens. Matter 19205105

[37] Donth E, Korus J, Hempel E and Beiner M 1997 Thermochim. Acta 305 239-249

[38] Wang L M, Velikov V and Angell C A 2002 J. Chem. Phys. 117 10184-1019

[39] Hecksher T, Olsen N B, Niss K and Dyre J C 2010 J. Chem. Phys. 133174514

[40] Wunderlich B (ed) 2005 Thermal analysis of polymeric materials (Springer-Verlag, Berlin Heidelberg)

[41] Fox T G and Flory P J 1950 J. Appl. Phys. 21 581-591

[42] Santangelo P G, Roland C M, Chang T, Cho D and Roovers J 2001 Macromolecules 34 9002-9005

[43] Ueberreiter K and Kanig G 1952 J. Coll. Sci. 7 569-583

[44] Johari G P and Goldstein M 1970 J. Chem. Phys. 53 2372-2388

[45] Ngai K L and Paluch M 2004 J. Chem. Phys. 120 857-873

[46] McCrum N G, Read B E and Williams G (eds) 1991 Anelastic and Dielectric Effects in Polymeric Solids (Dover Publications Inc., New York)

[47] Larini L, Ottochian A, Michele C D and Leporini D 2008 Nat. Phys. 4 42-45

[48] Scopigno T, Ruocco G, Sette F and Monaco G 2003 Science 302 849-852

[49] Utracki L A and Jamieson A M 2010 Polymer Physics: From Suspensions to Nanocomposites and Beyond (John Wiley Sons, Inc, Hoboken)

[50] Reading M and Hourtson D J 2006 Modulated Temperature Differential Scanning Calorimetry: Theoretical and Practical Applications in Polymer Characterisation (Springer, BerlinHeidelberg-New York)

[51] Pyda M, Van Durme K, Wunderlich B and Van Mele B 2005 J. Polym. Sci., Part B: Polym. Phys. 43 2141-2153

[52] Cangialosi D, Schwartz G A, Alegría A and Colmenero J 2005 J. Chem. Phys. 123144908

[53] Hodge I M 1994 J. Non-Cryst. Sol. 169 211-266

[54] Cangialosi D, Boucher V M, Alegría A and Colmenero J 2013 Phys. Rev. Lett. 111095701

[55] Priestley R D 2009 Soft Matter 5 919-926

[56] Kremer F and Schonhals A (eds) 2003 Broadband dielectric spectroscopy (Springer-Verlag, Berlin- 
Heidelberg)

[57] McCrum N G, Read B E and Williams G (eds) 1991 Anelastic and Dielectric Effects in Polymeric Solids (Dover Publications Inc., New York)

[58] Birge N O and Nagel S R 1985 Phys. Rev. Lett. 54(25) 2674-2677

[59] Schick C and Donth E 1991 Phys. Scr. 43 423-429

[60] Tyagi M, Alegría A and Colmenero J 2007 Phys. Rev. E 75061805

[61] Alvarez F, Alegría A and Colmenero J 1991 Phys. Rev. B 44 7306-7312

[62] Huth H, Minakov A A and Schick C 2006 J. Polym. Sci. Pt. B-Polym. Phys. 44 2996-3005

[63] Yan Y, Cheng L T and Nelson K A 1988 J. Chem. Phys. 88 6477-6486

[64] Christensen T and Olsen N B 1994 Phys. Rev. B 49(21) 15396-15399

[65] Fischer E W 1993 Physica A 201 183-206

[66] Richter D, Monkenbusch M, Arbe A and Colmenero J 2005 Neutron spin echo in polymer systems Neutron Spin Echo in Polymer Systems (Adv. Polym. Sci. vol 174) pp 1-221

[67] Colmenero J and Arbe A 2013 J. Polym. Sci. Pt. B-Polym. Phys. 51 87-113

[68] Ruta B, Chushkin Y, Monaco G, Cipelletti L, Pineda E, Bruna P, Giordano V M and GonzalezSilveira M 2012 Phys. Rev. Lett. 109165701

[69] Ruta B, Baldi G, Monaco G and Chushkin Y 2013 J. Chem. Phys. 138054508

[70] Simon S L, Sobieski J W and Plazek D J 2001 Polymer 42 2555-2567

[71] Boucher V M, Cangialosi D, Alegría A and Colmenero J 2011 Macromolecules 44 8333-8342

[72] Shi X F and McKenna G B 2005 Phys. Rev. Lett. 94157801

[73] Götze W and Sjogren L 1992 Rep. Prog. Phys. 55 241-376

[74] Garrahan J P and Chandler D 2002 Phys. Rev. Lett. 89035704

[75] Dyre J C 2006 Rev. Mod. Phys. 78 953-972

[76] Doolittle A K 1951 J. Appl. Phys. 22 1471-1475

[77] Cohen M H and Turnbull D 1959 J. Chem. Phys. 31 1164-1169

[78] Turnbull D and Cohen M H 1961 J. Chem. Phys. 34 120-125

[79] Goldstein M 1973 J. Phys. Chem. 77 667-673

[80] Corezzi S, Capaccioli S, Casalini R, Fioretto D, Paluch M and Rolla P 2000 Chem. Phys. Lett. $320113-117$

[81] Ferrer M, Lawrence C, Demirjian B, Kivelson D, Alba-Simionesco C and Tarjus G 1998 J. Chem. Phys. 109 8010-8015

[82] Roland C, Hensel-Bielowka S, Paluch M and Casalini R 2005 Rep. Prog. Phys. 68 1405-1478

[83] Roland C M 2010 Macromolecules 43 7875-7890

[84] Grassia L, Pastore Carbone M G and D'Amore A 2011 J. Appl. Polym. Sci. 122 3752-3757

[85] Gibbs J H and DiMarzio E A 1958 J. Chem. Phys. 28 373-383

[86] Adam G and Gibbs J H 1965 J. Chem. Phys. 43 139-146

[87] Matsuoka S (ed) 1992 Relaxation Phenomena in Polymers (Hnaser, New-York)

[88] Al Solunov C 2002 J. Phys. Condens. Matter 14 7297-7309

[89] Lubchenko V and Wolynes P G 2003 J. Chem. Phys. 119 9088-9105

[90] Donth E 1982 J. Non.Cryst. Sol. 53 325-330

[91] Hempel E, Hempel G, Hensel A, Schick C and Donth E 2000 J. Phys. Chem. B 104 2460-2466

[92] Saiter A, Delbreilh L, Couderc H, Arabeche K, Schoenhals A and Saiter J M 2010 Phys. Rev. E 81041805

[93] Donati C, Douglas J, Kob W, Plimpton S, Poole P and Glotzer S 1998 Phys. Rev. Lett. 80 $2338-2341$

[94] Berthier L, Biroli G, Bouchaud J P, Cipelletti L, Masri D E, L'Hôte D, Ladieu F and Pierno M 2005 Science 310 1797-1800

[95] Cangialosi D, Alegría A and Colmenero J 2007 Phys. Rev. E 76011514

[96] Gebremichael Y, Vogel M, Bergroth M, Starr F and Glotzer S 2005 J. Phys. Chem. B 109 15068-15079

[97] Dalle-Ferrier C, Thibierge C, Alba-Simionesco C, Berthier L, Biroli G, Bouchaud J P, Ladieu F, 
L'Hôte D and Tarjus G 2007 Phys. Rev. E 76041510

[98] Busch R, Liu W and Johnson W L 1998 J. Appl. Phys. 83 4134-4141

[99] Gallino I, Schroers J and Busch R 2010 J. Appl. Phys. 108063501

[100] Sastry S 2001 Nature 409 164-167

[101] Mossa S, La Nave E, Stanley H E, Donati C, Sciortino F and Tartaglia P 2002 Phys. Rev. E 65 041205

[102] Goldstein M 1976 J. Chem. Phys. 64 4767-4774

[103] Gujrati P D and Goldstein M 1980 J. Phys. Chem. 84 859-863

[104] Johari G P 2000 J. Chem. Phys. 112 7518-7523

[105] Dudowicz J, Freed K F and Douglas J F 2008 Generalized entropy theory of polymer glass formation Adv. Chem. Phys. (Adv. Chem. Phys. vol 137) ed Rice, SA pp 125-222

[106] Richert R and Angell C 1998 J. Chem. Phys. 108 9016-9026

[107] Cangialosi D, Alegría A and Colmenero J 2006 Macromolecules 39 7149-7156

[108] Schwartz G A, Colmenero J and Alegría A 2007 Macromolecules 40 3246-3255

[109] Cangialosi D, Alegría A and Colmenero J 2007 J. Chem. Phys. 126204904

[110] Prevosto D, Lucchesi M, Capaccioli S, Casalini R and Rolla P 2003 Phys. Rev. B 67174202

[111] Prevosto D, Capaccioli S, Lucchesi M, Leporini D and Rolla P 2004 J. Phys. Condens. Matt. 16 $6597-6608$

[112] Schwartz G A, Tellechea E, Colmenero J and Alegría A 2005 J. Non-Cryst. Solids 351 2616-2621

[113] Corezzi S, Fioretto D and Rolla P 2002 Nature 420 653-656

[114] Fan G J, Lavernia E J, Wunderlich R K and Fecht H J 2004 Philos. Mag. 84 2471-2484

[115] Pillips W, Buchenau U, Nucker N, Dianoux A and Petry W 1989 Phys. Rev. Lett. 63 2381-2384

[116] Angell C A and Borick S 2002 J. Non-Cryst. Solids 307 393-406

[117] Corezzi S, Comez L and Fioretto D 2004 Eur. Phys. J. E 14 143-147

[118] Richert R 2008 AIP Conf. Proc. 1027 1297-1299

[119] Angell C A 1997 J. Res. Natl. Inst. Stand. Technol. 102 171-185

[120] Ito K, Moynihan C T and Angell C A 1999 Nature 398 492-495

[121] Martinez L and Angell C 2001 Nature 410 663-667

[122] Wang L M, Angell C A and Richert R 2006 J. Chem. Phys. 125074505

[123] Ngai K L and Yamamuro O 1999 J. Chem. Phys. 111 10403-10406

[124] Santangelo P G and Roland C M 1998 Phys. Rev. B 58 14121-14123

[125] Roland C M, Santangelo P G and Ngai K L 1999 J. Chem. Phys. 111 5593-5598

[126] Huang D and McKenna G B 2001 J. Chem. Phys. 114 5621-5630

[127] Mohanty U, Craig N and Fourkas J 2001 J. Chem .Phys. 114 10577-10578

[128] Ruocco G, Sciortino F, Zamponi F, De Michele C and Scopigno T 2004 J. Chem. Phys. 120 10666

[129] Cangialosi D, Alegría A and Colmenero J 2006 J. Chem. Phys. 124024906

[130] Cangialosi D, Alegría A and Colmenero J 2005 Europhys. Lett. 70 614-620

[131] Tombari E, Ferrari C, Salvetti G and Johari G P 2008 Phys. Rev. B 78(14) 144203

[132] Cangialosi D, Alegría A and Colmenero J 2008 Phys. Rev. B 78(17) 176301

[133] Speedy R 1999 J. Phys. Chem. B 103 4060-4065

[134] Sokolov A P, Rössler E, Kisliuk A and Quittman D 1993 Phys. Rev. Lett. 71 2062-2065

[135] Novikov V N and Sokolov A P 2004 Nature 431 961-963

[136] Casalini R, Paluch M, Psurek T and Roland C M 2004 J. Mol. Liq. 111 53-60

[137] Johari G P 2006 Philos. Mag. 86 1567-1579

[138] Buchenau U and Wischnewski A 2004 Phys. Rev. B 70092201

[139] Scopigno T, Cangialosi D and Ruocco G 2010 Phys. Rev. B 81 100202(R)

[140] Niss K, Dalle-Ferrier C, Giordano V M, Monaco G, Frick B and Alba-Simionesco C 2008 J. Chem. Phys. 129194513

[141] Paluch M, Pawlus S, Hensel-Bielowka S, Kaminska E, Prevosto D, Capaccioli S, Rolla P A and Ngai K L 2005 J. Chem. Phys. 122234506 
[142] Ruta B, Baldi G, Scarponi F, Fioretto D, Giordano V M and Monaco G 2012 J. Chem. Phys. 137214502

[143] Yardimci H and Leheny R 2006 J. Chem. Phys. 124214503

[144] Dalle-Ferrier C, Niss K, Sokolov A P, Frick B, Serrano J and Alba-Simionesco C 2010 Macromolecules 43 8977-8984

[145] Hintermeyer J, Herrmann A, Kahlau R, Goiceanu C and Roessler E A 2008 Macromolecules 41 9335-9344

[146] Krygier E, Lin G X, Mendes J, Mukandela G, Azar D, Jones A A, Pathak J A, Colby R H, Kumar S K, Floudas G, Krishnamoorti R and Faust R 2005 Macromolecules 38 7721-7729

[147] Paluch M, Pawlus S, Sokolov A P and Ngai K L 2010 Macromolecules 43 3103-3106

[148] Wu J, Huang G, Wang X, He X and Zheng J 2011 Soft Matter 7 9224-9230

[149] Royal J S and Torkelson J M 1993 Macromolecules 26 5331-5335

[150] Dhinojwala A, Wong G K and Torkelson J M 1994 J. Chem. Phys. 100 6046-6054

[151] Alegría A, Guerrica-Echevarría E, Tellería I and Colmenero J 1993 Phys. Rev. B 47(22) 1485714865

[152] Chen K and Vyazovkin S 2009 J. Phys. Chem. B 113 4631-4635

[153] Sato K and Sprengel W 2012 J. Chem. Phys. 137104906

[154] Wojnarowska Z, Roland C M, Kolodziejczyk K, Swiety-Pospiech A, Grzybowska K and Paluch M 2012 J. Phys. Chem. Lett. 3 1238-1241

[155] Paluch M, Wojnarowska Z and Hensel-Bielowka S 2013 Phys. Rev. Lett. 110015702

[156] Nozaki R and Mashimo S 1987 J. Chem. Phys. 87 2271-2277

[157] O'Connell P A and McKenna G B 1999 J. Chem. Phys. 110 11054-11060

[158] Zhao J and McKenna G B 2012 J. Chem. Phys. 136154901

[159] Thurau C T and Ediger M D 2003 J. Chem. Phys. 118 1996-2004

[160] Cangialosi D, Wübbenhorst M, Groenewold J, Mendes E, Schut H, van Veen A and Picken S J 2004 Phys. Rev. B 70(22) 224213

[161] Zhao J, Simon S L and McKenna G B 2013 Nat. Commun. 41783

[162] Kruger J K, Britz T, Baller J, Possart W and Neurohr H 2002 Phys. Rev. Lett. 89285701

[163] Rivera A, Blochowicz T, Porokhonskyy V and Rossler E 2005 Phys. Rev. Lett. 94129603

[164] Welch R C, Smith J R, Potuzak M, Guo X, Bowden B F, Kiczenski T J, Allan D C, King E A, Ellison A J and Mauro J C 2013 Phys. Rev. Lett. 110(26) 265901

[165] Hecksher T, Nielsen A I, Olsen N B and Dyre J C 2008 Nat. Phys. 4 737-741

[166] Lunkenheimer P, Kastner S, Koehler M and Loidl A 2010 Phys. Rev. E 81051504

[167] Alegría A, Guerrica-Echevarria E, Goitiandia L, Telleria I and Colmenero J 1995 Macromolecules 28 1516-1527

[168] Wagner H and Richert R 1997 Polymer 38 255-261

[169] Richert R 2000 Physica A 287 26-36

[170] Boucher V M, Cangialosi D, Alegría A and Colmenero J 2012 Phys. Rev. E 86041501

[171] Richert R 2013 J. Chem. Phys. 139137101

[172] Zhao J and McKenna G B 2013 J. Chem. Phys. 139137102

[173] Liu L, Chen S, Faraone A, Yen C and Mou C 2005 Phys. Rev. Lett. 95117802

[174] Swenson J 2006 Phys. Rev. Lett. 97189801

[175] Cerveny S, Colmenero J and Alegria A 2006 Phys. Rev. Lett. 97189802

[176] Vogel M 2008 Phys. Rev. Lett. 101225701

[177] Doster W, Busch S, Gaspar A M, Appavou M S, Wuttke J and Scheer H 2010 Phys. Rev. Lett. 104098101

[178] Nakanishi M, Griffin P, Mamontov E and Sokolov A P 2012 J. Chem. Phys. 136124512

[179] Monasterio M, Jansson H, Gaitero J J, Dolado J S and Cerveny S 2013 J. Chem. Phys. 139 164714

[180] Avramov I and Milchev A 1988 J. Non-Cryst. Solids 104 253-260

[181] Di Marzio E A and Yang A J M 1997 J. Res. Natl. Inst. Stand. Technol. 102 135-157 
[182] Schweizer K S and Saltzman E J 2003 J. Chem. Phys. 119 1181-1196

[183] Chen K and Schweizer K S 2007 Phys. Rev. Lett. 98167802

[184] Elmatad Y S, Chandler D and Garrahan J P 2009 J. Phys. Chem. B 113 5563-5567

[185] Mauro J C, Allan D C and Potuzak M 2009 Phys. Rev. B 80094204

[186] Mauro J C, Yue Y, Ellison A J, Gupta P K and Allan D C 2009 Proc. Natl. Acad. Sci. U. S. A. 106 19780-19784

[187] Wang L, Li J and Fecht H J 2010 J. Phys.: Condens. Matter 22455104

[188] Kivelson D, Kivelson S, Zhao X, Nussinov Z and Tarjus G 1995 Physica A 219 27-38

[189] Kovacs A J, Aklonis J J, Hutchinson J M and Ramos A R 1979 J. Polym. Sci. Pt. B-Polym. Phys. 17 1097-1162

[190] Grassia L and Simon S L 2012 Polymer 53 3613-3620

[191] Hall R W and Wolynes P G 1987 J. Chem. Phys. 86 2943-2948

[192] Lubchenko V and Wolynes P G 2007 Annu. Rev. Phys. Chem. 58 235-266

[193] Bouchaud J and Biroli G 2004 J. Chem. Phys. 121 7347-7354

[194] Tanaka H, Kawasaki T, Shintani H and Watanabe K 2010 Nat. Mater. 9 324-331

[195] Parisi G and Zamponi F 2010 Rev. Mod. Phys. 82 789-845

[196] Bauwens-Crowet C and Bauwens J 1986 Polymer 27 709-713

[197] Brunacci A, Cowie J M G, Ferguson R and McEwen I J 1997 Polymer 38 3263-3268

[198] McGonigle E A, Cowie J M G, Arrighi V and Pethrick R A 2005 J. Mater. Sci. 40 1869-1881

[199] Cangialosi D, Boucher V M, Alegría A and Colmenero J $2013 \mathrm{~J}$. Polym. Sci., Part B: Polym. Phys. 51 847-853

[200] Cowie J M G, Harris S and McEwen I J 1997 J. Polym. Sci., Part B: Polym. Phys. 35 1107-1116

[201] Duenas J M M, Garayo A V, Colomer F R, Estelles J M, Ribelles J L G and Pradas M M 1997 J. Polym. Sci., Part B: Polym. Phys. 35 2201-2217

[202] Andreozzi L, Faetti M, Giordano M and Palazzuoli D 2003 J. Non-Cryst. Solids 332 229-241

[203] Koh Y P and Simon S L 2013 Macromolecules 46 5815-5821

[204] Cangialosi D, Alegría A and Colmenero J - Submitted - -

[205] Wolfgardt M, Baschnagel J, Paul W and Binder K 1996 Phys. Rev. E 54 1535-1543

[206] Johari G P 2000 J. Chem. Phys. 113 751-761

[207] Johari G P 2001 Chem. Phys. 265 217-231

[208] Johari G P 2001 J. Phys. Chem. B 105 3600-3604

[209] Pyda M and Wunderlich B 2002 J. Polym. Sci. Pt. B-Polym. Phys. 40 1245-1253

[210] WimbergerFriedl R and deBruin J G 1996 Macromolecules 29 4992-4997

[211] Robertson C G and Wilkes G L 2000 Macromolecules 33 3954-3955

[212] Golovchak R, Kozdras A, Balitska V and Shpotyuk O 2012 J. Phys. Condens. Matter 24505106

[213] Miller R and MacPhail R A 1997 J. Chem. Phys. 106 3393-3401

[214] Berens A R and Hodge I M 1982 Macromolecules 15 756-761

[215] Hodge I M and Berens A R 1982 Macromolecules 15 762-770

[216] Chen H, Inoue A and Masumoto T 1985 J. Mater. Sci. 20 2417-2438

[217] Boucher V M, Cangialosi D, Alegría A and Colmenero J 2010 Macromolecules 43 7594-7603

[218] Narayanaswamy O S 1971 J. Am. Ceram. Soc. 54 491-498

[219] Moynihan C T, Macedo P B, Montrose C J, Gupta P K, De Bolt M A, Dill J F, Dom B E, Drake P W, Eastel A J, Elterman P B, Moeller R P, Sasabe H and Wilder J A 1976 Ann. NY Acad.Sci. 279 15-35

[220] Lubchenko V and Wolynes P G 2004 J. Chem. Phys. 121 2852-2865

[221] Wisitsorasak A and Wolynes P G Cond. Matt. arXiv:1401.4485

[222] Grassia L Private Communication

[223] Swallen S F, Kearns K L, Mapes M K, Kim Y S, McMahon R J, Ediger M D, Wu T, Yu L and Satija S 2007 Science 315 353-356

[224] Leon-Gutierrez E, Sepulveda A, Garcia G, Teresa Clavaguera-Mora M and Rodriguez-Viejo J 2010 Phys. Chem. Chem. Phys. 12 14693-14698 
[225] Capponi S, Napolitano S and Wuebbenhorst M 2012 Nat. Commun. 31233

[226] Dawson K J, Kearns K L, Yu L, Steffen W and Ediger M D 2009 Proc. Natl. Acad. Sci. U.S.A. 106 15165-15170

[227] Guo Y, Morozov A, Schneider D, Chung J, Zhang C, Waldmann M, Yao N, Fytas G, Arnold C B and Priestley R D 2012 Nat. Mater. 11 337-343

[228] Jackson C L and McKenna G B 1991 J. Non-Cryst. Solids 131 221-224

[229] Keddie J L, Jones R A L and Cory R A 1994 Europhys. Lett. 27 59-64

[230] Forrest J A and Dalnoki-Veress K 2001 Adv. Colloid Interface Sci. 94 167-196

[231] Roth C and Dutcher J 2005 J. Electroanal. Chem. 584 13-22

[232] Alcoutlabi M and McKenna G B 2005 J. Phys.: Condens. Matter 17 R461-R524

[233] Tress M, Erber M, Mapesa E U, Huth H, Mueller J, Serghei A, Schick C, Eichhorn K J, Volt B and Kremer F 2010 Macromolecules 43 9937-9944

[234] Paeng K, Swallen S F and Ediger M D 2011 J. Am. Chem. Soc. 133 8444-8447

[235] Napolitano S and Wübbenhorst M 2011 Nat. Commun. 2260

[236] Boucher V M, Cangialosi D, Yin H, Schoenhals A, Alegría A and Colmenero J 2012 Soft Matter 8 5119-5122

[237] Zhang C, Boucher V M, Cangialosi D and Priestley R D 2013 Polymer 54230 - 235

[238] White R P and Lipson J E G 2011 Phs. Rev. E 84041801

[239] Forrest J A, Dalnoki-Veress K, Stevens J R and Dutcher J R 1996 Phys. Rev. Lett. 77 2002-2005

[240] Dalnoki-Veress K, Forrest J A, Murray C, Gigault C and Dutcher J R 2001 Phys. Rev. E 63 031801

[241] Roth C and Dutcher J 2003 Eur. Phys. J. E 12 S103-S107

[242] Liem H, Cabanillas-Gonzalez J, Etchegoin P and Bradley D 2004 J. Phys. Condens. Matter 16 $721-728$

[243] Miyazaki T, Inoue R, Nishida K and Kanaya T 2007 Eur. Phys. J. Spec. Top. 141 203-206

[244] Kim S, Roth C B and Torkelson J M 2008 J. Polym. Sci. Pt. B-Polym. Phys. 46 2754-2764

[245] Pye J E and Roth C B 2011 Phys. Rev. Lett. 107235701

[246] Baeumchen O, McGraw J D, Forrest J A and Dalnoki-Veress K 2012 Phys. Rev. Lett. 109055701

[247] Pye J E, Rohald K A, Baker E A and Roth C B 2010 Macromolecules 43 8296-8303

[248] Boucher V M, Cangialosi D, Alegría A and Colmenero J 2012 Macromolecules 45 5296-5306

[249] Napolitano S and Wuebbenhorst M 2010 Polymer 51 5309-5312

[250] Chowdhury M, Freyberg P, Ziebert F, Yang A C M, Steiner U and Reiter G 2012 Phys. Rev. Lett. 109136102 